\title{
Quantifying and Verifying Reachability for Access Controlled Networks
}

\author{
Alex X. Liu, Member, IEEE, and Amir R. Khakpour
}

\begin{abstract}
Quantifying and querying network reachability is important for security monitoring and auditing as well as many aspects of network management such as troubleshooting, maintenance, and design. Although attempts to model network reachability have been made, feasible solutions to computing network reachability have remained unknown. In this paper, we propose a suite of algorithms for quantifying reachability based on network configurations [mainly Access Control Lists (ACLs)] as well as solutions for querying network reachability. We present a network reachability model that considers connectionless and connection-oriented transport protocols, stateless and stateful routers/ firewalls, static and dynamic NAT, PAT, IP tunneling, etc. We implemented the algorithms in our network reachability tool called Quarnet and conducted experiments on a university network. Experimental results show that the offline computation of reachability matrices takes a few hours, and the online processing of a reachability query takes $0.075 \mathrm{~s}$ on average.
\end{abstract}

Index Terms-Algorithms, configuration, network reachability.

\section{INTRODUCTION}

\section{A. Motivation}

$\mathbf{T}$ HIS paper concerns quantifying and verifying reachability between any two subnets in an interconnected and access controlled network. The network reachability refers to the set of packets that can traverse from one subnet to another, based on network configurations. Although computer networks are created to provide reachability between end-hosts, Access Control Lists (ACLs) on routers and firewalls are commonly used to limit such reachability for security or privacy purposes, and various factors, such as routing dynamics and network address translation, may affect such reachability, in unexpected ways. As a critical infrastructure of national importance, the Internet faces unprecedent challenges for reliable reachability. Correctly implementing the exact reachability that is needed for a large interconnected network is crucial. While more reachability than necessary may open doors to unwanted and even malicious traffic causing sometimes irreparable damages,

Manuscript received July 09, 2010; revised August 31, 2011 and April 12, 2012; accepted May 29, 2012; approved by IEEE/ACM TRANSACTIONS ON NETWORKING Editor A. Feldmann. Date of publication July 23, 2012; date of current version April 12, 2013. This work was supported in part by the National Science Foundation under Grants CNS-0716407, CNS-0916044, and CNS-0845513. The preliminary version of this paper, titled "Quantifying and Querying Network Reachability," was published in the Proceedings of the 30th International Conference on Distributed Computing Systems (ICDCS), Genoa, Italy, June 21-25, 2010. A. X. Liu is the corresponding author of this paper.

The authors are with the Department of Computer Science and Engineering, Michigan State University, East Lansing, MI 48824 USA (e-mail: alexliu@cse. msu.edu; khakpour@cse.msu.edu).

Color versions of one or more of the figures in this paper are available online at $\mathrm{http}: / /$ ieeexplore.ieee.org.

Digital Object Identifier 10.1109/TNET.2012.2203144 less reachability than necessary may disrupt normal businesses causing huge revenue losses.

Unfortunately, in reality, network reachability management is often a mess for many networks due to its high complexity and the lack of advanced reachability debugging tools. First, network configurations have become far more complex than even skilled operators can correctly manage. The complexity of modern networks has been rapidly increasing due to the explosive growth of Internet connectivity expanding from end-hosts to pervasive devices and network-supported applications of various scales. Second, due to the lack of advanced reachability debugging tools, the current common practice for reachability management is still "trial and error," which of course results in a plethora of reachability errors. Configuration errors have been observed to be the largest cause of failure for Internet services [1]. A recent Yankee Group report has shown that more than $62 \%$ of network downtime is due to human configuration errors, and more than $80 \%$ of IT budgets are allocated toward maintaining just the status quo [2]. Network operators face tremendous pressure to fix problems quickly because operational networks often support critical business applications and important communications, and the loss caused by network outrages becomes increasingly acute. For example, the estimated revenue losses per hour of downtime for the industry of media, banking, and brokerage are $\$ 1.2, \$ 2.6$, and $\$ 4.5$ million, respectively [2]. The reality could be much worse than these published staggering numbers as the errors of more reachability than needed are often go undetected, while less reachability than needed is a common source of complaints to operators. Therefore, a scientific approach, instead of "trial and error," is needed to manage network reachability.

As organizations continually transform their network infrastructure to maintain their competitive edge by deploying new servers, installing new software and services, expanding connectivity, etc., operators constantly need to manually modify their network configurations. Ideally, such manual modifications should exactly implement the desired changes. However, practically, they often come with undesirable, yet unnoticed, side effects on reachability. Therefore, an automated tool that monitors the all-to-all reachability of a large interconnected network in real time is crucial to its successful management. Furthermore, querying and verifying reachability is a routine, yet error-prone, task for operators. An automated reachability query and verification tool is needed.

To enforce the right amount of network reachability, no more and no less, for a large interconnected enterprise network to achieve access control, security, and privacy, in this paper, we investigate two aspects of network reachability - quantification and querying - and present a reachability management tool, which we call Quarnet. Quantifying the reachability between any two subnets means to compute the set of packets that 
can traverse from one subnet to another based on network configurations. Querying reachability means to ask questions like "who can access what." They are useful in many aspects of network management

1) Network Security Monitoring and Auditing: Verifying that the deployed ACLs satisfy certain security specifications is an integral part of network security monitoring and auditing. The current practice is to send probing packets. However, this approach has drawbacks. First, it is infeasible to generate all possible probing packets. Second, as routing tables change over time, the auditing result is valid only for a specific time. Combining the current active probing approach with quantitative network reachability analysis, we can have comprehensive security auditing for large enterprise networks.

2) Network Troubleshooting: An important task for network administrators is to troubleshoot reachability problems when two hosts fail to communicate or there is unauthorized traffic passing through a series of ACLs. For large complex networks, troubleshooting reachability problems is extremely difficult. To check the reachability of a path, the current practice is to check the reachability of every hop in the path by actively sending probing packets. This approach is disruptive, time-consuming, and sometimes infeasible when isolating some hops is impossible. In contrast, when the reachability of every path has been precomputed, troubleshooting reachability problems and identifying faulty ACLs are easy.

3) Network Security Design, Maintenance, and Reachability Monitoring: Designing ACLs based on certain security policies is an important part of network design. Before deploying a network, it is important to first verify reachability. This helps to avoid security breaches and service outbreaks caused by misconfigured ACLs. Furthermore, networks change over time with the evolving of topology and connected servers/hosts. Often, network changes require corresponding changes on ACL rules. Due to the interconnected nature of networks, a modification on one ACL may have unnoticed side effects on network reachability such as causing two servers to fail to communicate or opening security holes by enabling unauthorized accesses. Thus, network reachability analysis helps to detect and resolve potential problems before committing any change to ACLs.

\section{B. Limitation of Prior Art}

Little work has been done on network reachability analysis. Xie et al. presented a model of network reachability in their seminal work [3], however they give no algorithms for computing reachability (and of course no experimental results). Xie et al.'s network reachability model does not address IP tunneling, dynamic NAT, and PAT and does not consider whether transportlayer protocols are connectionless or connection-oriented. Furthermore, Xie et al.'s model is limited to describing the networks where each subnet connects to only one router because they model a network as a graph over only routers.

\section{Technical Challenges}

Computing network reachability is hard. First, from the reachability perspective, the interaction among the rules in one ACL is already complex due to the multidimensionality of ACL rules, but the interaction of multiple ACLs in interconnected networks is even more complex. Second, routing and network topology have complex impact on reachability.

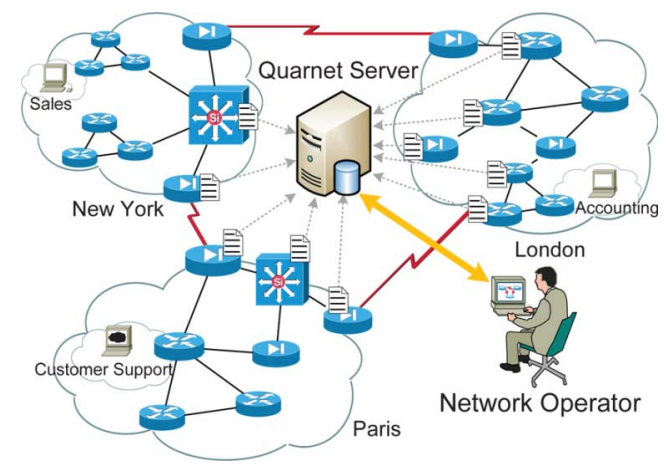

Fig. 1. Quarnet architecture.

There is typically more than one path between a source and a destination, and a packet may only be able to traverse from the source to the destination via some, but not all, available paths. Third, middleboxes often have complex impact on reachability. For example, packet transforming middleboxes [such as Network Address Translation (NAT) and Port Address Translation (PAT)] and IP tunneling complicate reachability calculation because they modify packets headers when they are traveling in a network. Fourth, transport-layer protocols also complicate reachability calculation because, for connection-oriented protocols, the reachability of both data path and signaling path should be taken into account. It is even more challenging while there are some stateful middleboxes in the path. Last but not least, the problem space is huge as the ACL rules are typically specified over the standard 5-tuple with 104 bits in total. It has been observed that the ACLs on the Internet often have errors [1], [3], [4]. An error in an ACL either creates security holes that will allow malicious traffic to sneak into a private network or blocks legitimate traffic and disrupts normal businesses, which in turn could lead to irreparable, if not tragic, consequences.

\section{Our Approach}

In this paper, we present Quarnet, a tool that comprises a suite of concrete algorithms for quantifying and querying network reachability. For quantification, Quarnet takes a network topology and the ACLs deployed on middleboxes as its input, and outputs reachability matrices that represent the lower-bound reachability (i.e., the minimal set of packets that can traverse from a source to a destination at any time), instantaneous reachability (i.e., the set of packets that can traverse from a source to a destination at a particular time), and upper-bound reachability (i.e., the maximal set of packets that can traverse from a source to a destination at some time) for every pair of source and destination subnets. For querying, Quarnet allows network operators to ask questions about the reachability of the subnets in their network, e.g., "which hosts in subnet A can access the mail server in subnet B?" We proposed a language for formally specifying reachability queries. To efficiently process queries, we use decision diagrams as the data structure for representing reachability matrices.

Quarnet can be deployed on a server as shown in Fig. 1. Initially, this server first collects the configuration file from each middlebox (via SNMP for example) and the network topology from the administrator and then computes reachability matrices offline. Afterwards, network operators can perform reachability 
queries through a GUI, where each query is then formulated by an SQL-like query language and processed by the Quarnet query module. Fine-grained access control to reachability matrices can be enforced so that different operators can perform different reachability queries. Each time the configuration of a middlebox or the topology of the network is changed, the Quarnet server needs to be notified and reachability matrices need to be updated accordingly.

The focus of this paper is on network reachability at layers 3 and 4 . We do not deal with packet filtering technologies, such as Intrusion Detection and Prevention Systems (IDSs/IPSs), which filter packets based on packet payload because packet payload filtering is orthogonal to packet header filtering. Layer-7 reachability is out of the scope of this paper. Furthermore, Quarnet is targeted at enterprise networks, and we assume there are no unauthorized or undocumented elements.

\section{E. Key Contributions}

We make four key contributions in this paper. 1) We propose a comprehensive reachability modeling and formulation that encompasses elements that have not been addressed in prior work such as dynamic NAT, PAT, IP tunneling, connection orientation of transport protocols, and statefulness of middleboxes. 2) We propose efficient algorithms for computing network reachability. 3) We propose a language and solutions for querying network reachability. 4) We implemented Quarnet in C++ and experimented on a university network. Experimental results show that the offline computation of reachability matrices takes $11 \mathrm{~h}$, and the online processing of a reachability query takes an average of $0.075 \mathrm{~s}$.

\section{REACHABILITY MODELING AND FORMULATION}

\section{A. Network Modeling}

In this paper, we model a network as a nonsimple directed bipartite graph $\mathcal{G}=(\mathcal{V}, \mathcal{E}, \mathcal{F})$, where $\mathcal{V}$ is a set of vertices, $\mathcal{E}$ is a set of arcs over $\mathcal{V}$, and $\mathcal{F}$ is a set of ACLs. Each vertex in $\mathcal{V}$ represents a subnet or a middlebox. We use the term "subnet" to represent a set of adjacent subnetworks [i.e., local area networks (LANs) or virtual LANs (VLANs)], where either they have the same reachability (i.e., there is no ACL deployed between any two subnetworks in the set) or the reachability among the subnetworks is not a concern. For example, given an enterprise network, we represent Internet as a subnet connected to the gateway router. The term "middlebox" refers to any networking device that can forward packets from one subnet to another, such as a network router, a firewall, a traffic shaper, or an L3 switch. We model a network as a bipartite graph because between two adjacent subnets there is at least one middlebox, and between any two middleboxes there exists at least one subnet. Let $\mathcal{N}$ be the set of subnets in $\mathcal{V}$ and $\mathcal{R}$ be the set of middleboxes in $\mathcal{V}$. Each arc in $\mathcal{E}$ represents a unidirectional physical link between a subset in $\mathcal{N}$ and a middlebox in $\mathcal{R}$. We model network links as unidirectional arcs because every ACL is associated with a unidirectional physical link and each bidirectional link can be modeled as two unidirectional arcs. Note that some physical links, such as satellite links, are physically unidirectional. We model a network as a nonsimple graph because between a subnet and a middlebox there may exist multiple physical links for backup. Each ACL in $\mathcal{F}$ filters the packets traveling on an arc in $\mathcal{E}$. Given a network with $m$ middleboxes, where the maximum number

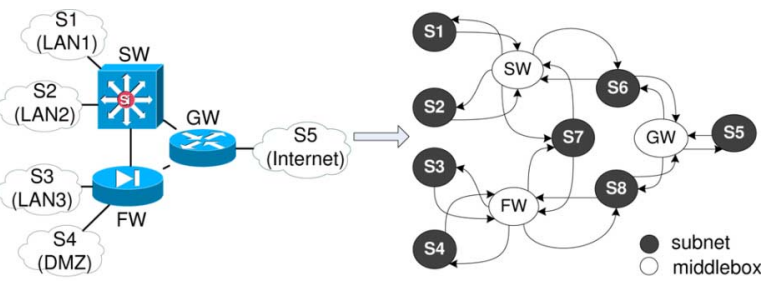

(a)

(b)

Fig. 2. Example network topology. (a) Network topology. (b) Network model.

of unidirectional interfaces on a middlebox is denoted $h$, we represent the network by an $m \times h$ matrix $\mathcal{I}$ called the Network Incident Matrix. Here, $\mathcal{I}[i, j]=x$ if and only if subnet $x \in \mathcal{N}$ connects to middlebox $i \in \mathcal{R}$ on its interface $j$, and $\mathcal{I}[i, j]=0$ if and only if no subnet connects to middlebox $i$ on its interface $j$. For simplicity, incoming interfaces are represented by even numbers, and outgoing interfaces are represented by odd numbers. Similarly, we represent the ACLs deployed on the network by an $m \times h$ matrix $\mathcal{A}$ called the $A C L$ Matrix. We use $\mathcal{A}[i, j]$ to denote the ACL deployed on the $j$ th interface of middlebox $i$.

Fig. 2(a) shows a network with three middleboxes and four subnetworks. Two VLANs (S1 and S2) are connected to an L3 switch (SW). One subnetwork (S3) and a DMZ (S4) are connected to the firewall (FW). SW and FW are connected to the Internet through a gateway router (GW). Fig. 2(b) shows graph representing the topology. Note that we assume there is an ACL on each interface of the middleboxes. The graph consists of 11 vertices representing the eight subnets $S 1, \ldots, S 8$ and the three middleboxes SW, FW, and GW. Note that $\mathrm{S} 1, \ldots, \mathrm{S} 4$ denote the four subnetworks LAN1, LAN2, LAN3, and DMZ, S5 denotes the outside Internet, and $S 6, \ldots, S 8$ denote the subnetworks that connects two adjacent middleboxes. The network incident matrix for this network with $m=3$ and $h=8$ is

$$
\mathcal{I}=\left(\begin{array}{cccccccc}
S 1 & S 1 & S 2 & S 2 & S 6 & S 6 & S 7 & S 7 \\
S 3 & S 3 & S 4 & S 4 & S 7 & S 7 & S 8 & S 8 \\
S 5 & S 5 & S 6 & S 6 & S 8 & S 8 & 0 & 0
\end{array}\right) .
$$

Between any two directly connected middleboxes, our model assumes there is a subnetwork because the middlebox interfaces may have a distinct IP address and an ACL guarding that interface. The reachability of such a subnetwork is important because of several reasons. First, there are often some management services on a middlebox (such as SNMP, Telnet, and SSH) that are accessible through each interface. Such services are intended to be used only by administrators. Therefore, there are often some rules in the ACL deployed on each interface to restrict the access to this subnetwork. Second, if a middlebox is compromised, the source of the subsequent attacks is the IP address of an interface of the middle box; thus, the reachability from that subnetwork to other subnetworks is critical. Indeed, if the interfaces are not assigned IP addresses, the subnet is modeled with an empty address set; henceforth, reachability to and from the subnetwork is empty.

Table I lists important notations used in this paper.

\section{B. Reachability Formulation}

Network reachability depends on not only some static factors, i.e., topology and ACL configurations, but also on some dynamic factors, i.e., routing states, where each is defined as a 
TABLE I

NOTATION TABLE

\begin{tabular}{|l|l|l|l|}
\hline \multicolumn{3}{|c|}{ Notation Table } \\
\hline $\mathcal{N}$ & set of subnets & $n$ & \# of subnets \\
$\mathcal{R}$ & set of middleboxes & $m$ & \# of middleboxes \\
$h$ & max \# of unidirectional & $\mathcal{I}$ & network incident matrix \\
& interfaces on a middlebox & $\mathcal{A}$ & ACL matrix \\
$p$ & number of the paths in a network & $s$ & routing state \\
$P_{i, j}(s)$ & path from $N_{i}$ to $N_{j}$ & $M$ & \# of hops on a path \\
$A\left(C_{k}\right)$ & at routing state $s$ & $C_{k}$ & $k$-th classifier in a path \\
$z$ & packets accepted by ACL $C_{k}$ & & from $N_{i}$ to $N_{j}$ \\
$F_{i}$ & max \# of paths between any & $R$ & reachability set \\
$f$ & two subnets & $\ell$ & ACL \\
$g$ & $i$-th field in FDD & $F$ & FDD matrix \\
$d$ & Firewall Decision Diagram (FDD) & $P$ & path matrix \\
& max \# of rules in an ACL & $N_{i}$ & subnet $i$ \\
\hline
\end{tabular}

snapshot of all the routing tables of the middleboxes in the network, and the one-to-one mapping tables of dynamic NATs and PATs. We formulate three types of network reachability: lowerbound reachability, instantaneous reachability, and upper-bound reachability for a given network topology and the ACL configurations. We define the lower-bound reachability from subnet $N_{i}$ to $N_{j}$ as the set of packets that can go from $N_{i}$ to $N_{j}$ at any time. We define the instantaneous reachability from subnet $N_{i}$ to $N_{j}$ as the set of packets that can go from $N_{i}$ to $N_{j}$ at a particular time. We define the upper-bound reachability from subnet $N_{i}$ to $N_{j}$ as the maximal set of packets that can go from $N_{i}$ to $N_{j}$ at some time.

Below, we formulate instantaneous, upper-bound, and lowerbound reachability. In our notations, we use "I," "U," and "L" to denote instantaneous, upper-bound, and lower-bound reachability, respectively; we further use "CL" and "CO" to denote connectionless and connection-oriented protocols.

In formulating network reachability, we differentiate connectionless transport protocols (e.g., UDP, ICMP) and connectionoriented transport protocols (e.g., TCP). In connectionless transport protocols, only the destination needs to be reachable from the source. However, in connection-oriented transport protocols, both the source and destination need to be reachable from each other to ensure the transmission of acknowledgment messages between them. For connection-oriented transport protocols, we further differentiate the following three cases based on the statefulness of the routers on the path from a source to a destination: 1) all routers are stateless; 2) all routers are stateful; and 3 ) some routers are stateless and some are stateful. For stateful routers, because they monitor connection states, they allow signaling packets associated with an existing connection to pass through and drop signaling packets that are not associated with any connection. For stateless routers, they match all signaling packets against their ACLs. Therefore, in calculating the reachability of stateless routers, we need to consider the impact of their ACLs on signaling packets.

1) Instantaneous Reachability: Given a network routing state $s$, which is a snapshot of all the routing tables of the middleboxes in the network, let $P_{i, j}(s)$ denote the path from $N_{i}$ to $N_{j}$ at state $s$, and $M$ denote the number of hops/middleboxes on path $P_{i, j}(s)$. For the $k$ th middlebox, we use $C_{2 k-1}$ to denote the ACL on the incoming middlebox interface, and $C_{2 k}$ to denote the ACL on the outgoing middlebox interface.

For connectionless protocols (mainly the UDP protocol), the instantaneous reachability from $N_{i}$ to $N_{j}$ is the intersection of the set of UDP packets accepted by every ACL on the path from $N_{i}$ to $N_{j}$. Thus, we calculated instantaneous reachability as follows:

$$
R_{\mathrm{CL}}^{\mathrm{I}}(i, j, s)=\bigcap_{k=1}^{2 M} A_{\mathrm{UDP}}\left(C_{k}\right)
$$

where $A_{\mathrm{UDP}}\left(C_{k}\right)$ is the set of UDP packets accepted by $C_{k}$.

For connection-oriented protocols (mainly the TCP protocol), the instantaneous reachability from $N_{i}$ to $N_{j}$ also depends on the reachability of the acknowledgment (ACK) messages from $N_{j}$ to $N_{i}$. To incorporate the signaling path reachability of data path $P_{i, j}(s)$, we distinguish the statefulness of the intermediate middleboxes according to the following three cases: all middleboxes in $P_{i, j}(s)$ are stateful, all middleboxes in $P_{i, j}(s)$ are stateless, and $P_{i, j}(s)$ contains both stateful middleboxes and stateless middleboxes.

All Middleboxes in $\mathbf{P}_{\mathbf{i}, \mathbf{j}}(\mathbf{s})$ Are Stateful: In any stateful middlebox on path $P_{i, j}(s)$, the state of every TCP session is stored in a state table to ensure that the corresponding signaling messages can traverse back from $N_{j}$ to $N_{i}$. Such messages are not checked against any ACL of the middleboxes on path $P_{j, i}(s)$. When a signaling message does not match any entry in the state table of a stateful middlebox, the message is dropped, and the connection will fail. Here, we assume that the network is designed such that we have path-coupled signaling on stateful firewalls and NAT, which means that the forward data path and the backward signaling path contain the same set of middleboxes. The path-coupled property holds for most existing networks [5], [6]. Thus, when all middleboxes in $P_{i, j}(s)$ are stateful, the instantaneous reachability from $N_{i}$ to $N_{j}$ is the intersection of the set of TCP packets accepted by every ACL on the path from $N_{i}$ to $N_{j}$. Therefore, we calculated instantaneous reachability for this case as follows, where $A_{\mathrm{TCP}}\left(C_{k}\right)$ represents the set of TCP packets accepted by ACL $C_{k}$ :

$$
R_{\mathrm{CO}}^{\mathrm{I}}(i, j, s)=\bigcap_{k=1}^{2 M} A_{\mathrm{TCP}}\left(C_{k}\right) .
$$

All Middleboxes in $\mathbf{P}_{\mathbf{i}, \mathbf{j}}(\mathbf{s})$ Are Stateless: If all the intermediate middleboxes are stateless, we not only need to consider the data path from $N_{i}$ to $N_{j}$, but also the signaling path from $N_{j}$ to $N_{i}$. Let $\tilde{A}$ represent the set of accepted packets where in each packet the values of source and destination IP address fields are swapped, and the values of source and destination port number fields are also swapped. Field swapping is needed because, in one TCP session, each data packet and its corresponding signaling packet have their IP addresses and port numbers in the opposite order. Note that when all middleboxes in $P_{i, j}(s)$ are stateless, we do not need path-coupled assumption. Thus, the instantaneous reachability for the connection-oriented and reliable protocols is the intersection of the set of accepted TCP packets in the data path and the set of accepted TCP packets in the signaling path. Therefore, we calculated instantaneous reachability for this case as follows, where the ACLs on path $P_{j, i}$ consist of $C_{1}^{\prime}, C_{2}^{\prime}, \ldots, C_{2 M^{\prime}}^{\prime}$ :

$$
R_{\mathrm{CO}}^{\mathrm{I}}(i, j, s)=\bigcap_{k=1}^{2 M} A_{\mathrm{TCP}}\left(C_{k}\right) \bigcap_{k=1}^{2 M^{\prime}} \tilde{A}_{\mathrm{TCP}}\left(C_{k}^{\prime}\right) .
$$


(Here, $\quad \bigcap_{k=1}^{2 M} A_{\mathrm{TCP}}\left(C_{k}\right) \bigcap_{k=1}^{2 M^{\prime}} A_{\mathrm{TCP}}\left(C_{k}^{\prime}\right) \quad$ denotes $A_{\mathrm{TCP}}\left(C_{1}\right) \cap \cdots \cap A_{\mathrm{TCP}}\left(C_{2 M}\right) \cap A_{\mathrm{TCP}}\left(C_{1}^{\prime}\right) 1 \cap \cdots \cap$ $A_{T \mathrm{CP}}\left(C_{2 M^{\prime}}^{\prime}\right)$.)

$\mathbf{P}_{\mathbf{i}, \mathbf{j}}(\mathbf{s})$ Contains Both Stateful Middleboxes and Stateless Middleboxes: For stateful middleboxes, we again need assumption of path-coupled signaling. For stateless middleboxes, we do not need this assumption. Thus, the instantaneous reachability on $P_{i, j}(s)$ is the intersection of the set of accepted packets of stateful middleboxes calculated by (2) and the set of accepted packets of stateless routers calculated by (3).

2) Reachability Bounds: The Reachability Lower Bound from $N_{i}$ to $N_{j}, R^{\mathrm{L}}(i, j)$, denotes the set of packets that can traverse from $N_{i}$ to $N_{j}$ in all routing states. The Reachability Upper Bound from $N_{i}$ to $N_{j}, R^{\mathrm{U}}(i, j)$, denotes the maximal set of packets that can traverse from $N_{i}$ to $N_{j}$ in some routing states. Let $\mathcal{S}$ denote the set of all routing states of a network. The reachability lower bound and upper bound from $N_{i}$ to $N_{j}$ is calculated as follows:

$$
\begin{aligned}
& R_{\mathrm{CL}}^{\mathrm{U}}(i, j)=\bigcup_{s \in \mathcal{S}} R_{\mathrm{CL}}^{\mathrm{I}}(i, j, s) \\
& R_{\mathrm{CL}}^{\mathrm{L}}(i, j)=\bigcap_{s \in \mathcal{S}} R_{\mathrm{CL}}^{\mathrm{I}}(i, j, s) .
\end{aligned}
$$

Similar to the reachability bounds for connectionless protocols, the reachability bounds of the connection-oriented protocols using (2) and (3) are calculated as follows:

$$
\begin{aligned}
& R_{\mathrm{CO}}^{\mathrm{U}}(i, j)=\bigcup_{s \in \mathcal{S}} R_{\mathrm{CO}}^{\mathrm{I}}(i, j, s) \\
& R_{\mathrm{CO}}^{\mathrm{L}}(i, j)=\bigcap_{s \in \mathcal{S}} R_{\mathrm{CO}}^{\mathrm{I}}(i, j, s) .
\end{aligned}
$$

Computing reachability lower bound and upper bound is very useful. For example, lower-bound reachability can be used to ensure that the available services on a subnet are reachable regardless of routing states, and upper-bound reachability can be used to ensure that the access to some services is restricted. Furthermore, the reachability upper bound and lower bound are useful in verifying the correctness of ACLs. Ideally, the reachability upper bound and lower bound from $N_{i}$ to $N_{j}$ should be the same (i.e., $\Delta R(i, j)=R^{\mathrm{U}}(i, j)-R^{\mathrm{L}}(i, j)$ should be $\left.\emptyset\right)$. Otherwise, the ACLs have inconsistent decisions for the packets in $\Delta R(i, j)$ : Sometimes they are allowed to traverse from $N_{i}$ to $N_{j}$, and sometimes they are not. For a packet $\pi \in \Delta R(i, j)$, if $\pi$ should be constantly allowed to traverse from $N_{i}$ to $N_{j}$, then blocking $\pi$ at some routing states may disrupt legitimate services; if $\pi$ should be constantly disallowed to traverse from $N_{i}$ to $N_{j}$, then accepting $\pi$ at some states may cause security breaches.

\section{Algorithms FOR COMPUting REACHABILITY MATRICES}

In this section, we present algorithms for computing reachability for networks with no packet transformation filters.

\section{A. Reachability Matrices}

We represent network reachability as the six matrices shown below. We use $n$ to denote the number of subnets, and $z$ to denote the maximum number of paths between any pair of subnets:

1) $A_{\mathrm{CL}}^{\mathrm{I}}[1 \ldots n, 1 \ldots n, 1 \ldots z]$, where each element $A_{\mathrm{CL}}^{\mathrm{I}}[i, j, k]$ is the set of packets representing the in- stantaneous reachability from $N_{i}$ to $N_{j}$ on the $k$ th path for connectionless protocols;

2) $A_{\mathrm{CO}}^{\mathrm{I}}[1 \ldots n, 1 \ldots n, 1 \ldots z, 1 \ldots z]$, where each element $A_{\mathrm{CO}}^{\mathrm{I}}\left[i, j, k, k^{\prime}\right]$ is the set of packets representing the instantaneous reachability from $N_{i}$ to $N_{j}$ on the $k$ th data path and the $k^{\prime}$ th signaling path for connection-oriented protocols;

3) $A_{\mathrm{CL}}^{\mathrm{L}}[1 \ldots n, 1 \ldots n]$, where each element $A_{\mathrm{CL}}^{\mathrm{L}}[i, j]$ is the set of packets representing the lower-bound reachability from $N_{i}$ to $N_{j}$ for connectionless protocols;

4) $A_{\mathrm{CO}}^{\mathrm{L}}[1 \ldots n, 1 \ldots n]$, where each element $A_{\mathrm{CO}}^{\mathrm{L}}[i, j]$ is the set of packets representing the lower-bound reachability from $N_{i}$ to $N_{j}$ for connection-oriented protocols;

5) $A_{\mathrm{CL}}^{\mathrm{U}}[1 \ldots n, 1 \ldots n]$, where each element $A_{\mathrm{CL}}^{\mathrm{U}}[i, j]$ is the set of packets representing the upper-bound reachability from $N_{i}$ to $N_{j}$ for connectionless protocols;

6) $A_{\mathrm{CO}}^{\mathrm{U}}[1 \ldots n, 1 \ldots n]$, where each element $A_{\mathrm{CO}}^{\mathrm{U}}[i, j]$ is the set of packets representing the upper-bound reachability from $N_{i}$ to $N_{j}$ for connection-oriented protocols.

\section{B. Basic Data Structures and Algorithms}

For any ACL $\ell$, we define the accept set of $\ell$, denoted $\operatorname{accept}(\ell)$, to be the set of packets that can be accepted by $\ell$. In this section, we first consider the following core problem in computing network reachability matrices: Given two ACLs $\ell_{1}$ and $\ell_{2}$, how can we compute $\operatorname{accept}\left(\ell_{1}\right) \cap \operatorname{accept}\left(\ell_{2}\right)$ and accept $\left(\ell_{1}\right) \cup \operatorname{accept}\left(\ell_{2}\right)$ ? Our algorithm for this computation consists of three steps: firewall decision diagram (FDD) construction, FDD shaping, and FDD logical operations.

1) Step I-FDD Construction: In this step, we convert each ACL to an equivalent FDD. FDD was introduced by Gouda and Liu in [7] as a data structure for representing access control lists. In a nutshell, an FDD is a decision tree where nodes are rule fields (or packet fields) and edges are labeled by a set of intervals. The leaves of the tree are the actions that have to be taken for the matched packet. As a formal definition, a firewall decision diagram with a decision set $D S$ and over fields $F_{1}, \ldots, F_{d}$ is an acyclic and directed graph that has the following five properties.

1) There is one root node that has no incoming edges. The nodes with no outgoing edges are called terminal nodes.

2) Each node $v$ has a label, denoted $F(v)$, such that $F(v) \in$ $\left\{F_{1}, \ldots, F_{d}\right\}$ if $v$ is a nonterminal node and $F(v) \in D S$ if $v$ is a terminal node.

3) Each edge $e: u \rightarrow v$ is labeled with a nonempty set of integers, denoted $I(e)$, where $I(e) \subseteq D(F(u))$.

4) A directed path from the root to a terminal node is called a decision path. No two nodes on a decision path have the same label.

5) The set of all outgoing edges of a node $v$, denoted $E(v)$, satisfies the following two conditions:

a) Consistency: $I(e) \cap I\left(e^{\prime}\right)=\emptyset$ for any two distinct edges $e$ and $e^{\prime}$ in $E(v)$.

b) Completeness: $\bigcup_{e \in E(v)} I(e)=D(F(v))$.

Decisions are $\{1,0\}$, where 1 represents accept and 0 represents discard. We call such FDDs "Binary FDDs." In converting an ACL to an equivalent binary FDD, we replace all flavors of accept, such as accept and accept with logging, by 1, and replace all flavors of discard, such as discard, reject, and discard/reject with logging, by 0 . We further define a full-length ordered FDD as an FDD where in each decision path, all fields appear exactly 


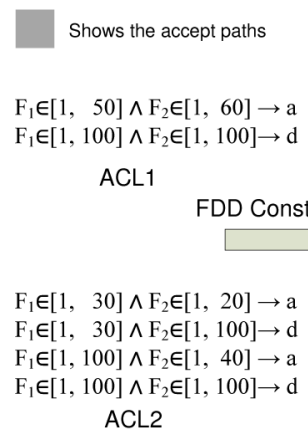

(a)
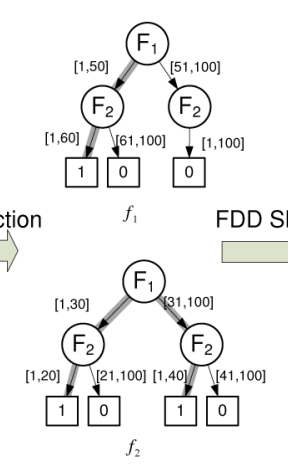

(b)

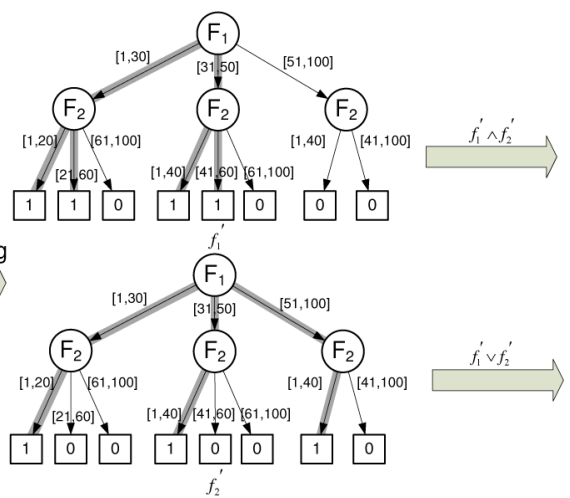

(c)
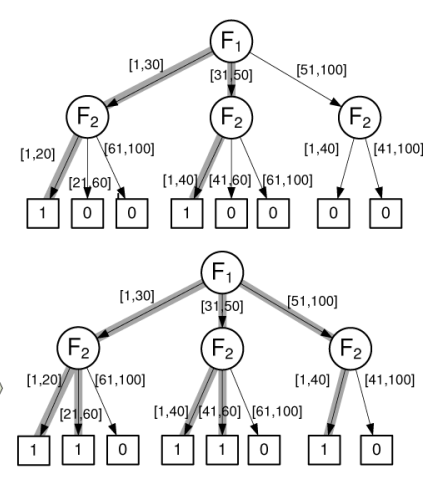

(d)

Fig. 3. (a) Two ACLs. (b) Two FDDs before shaping. (c) Two FDDs after shaping. (d) FDD logical AND/OR.

once and in the same order. For ease of presentation, in the rest of this paper, we use the term "FDD" to mean "binary full-length ordered FDD" if not otherwise specified. Fig. 3(b) shows the two FDDs constructed from the two ACLs in Fig. 3(a).

An FDD construction algorithm, which converts a sequence of range rules to an equivalent full-length ordered FDD, is described in [8]. For computing reachability matrices, we choose the protocol type field as the label of the root node.

We call the decision paths whose terminal nodes are labeled 1 accept paths. Similarly, we call the decision paths whose terminal nodes are labeled 0 discard paths. Given an ACL $\ell$, after we convert it to an equivalent FDD $f$, the accept paths of $f$ represent the set accept $(\ell)$.

2) Step II-FDD Shaping: In the previous step, we convert the two given ACLs $\ell_{1}$ and $\ell_{2}$ to two FDDs $f_{1}$ and $f_{2}$ such that $\ell_{1}$ is equivalent to $f_{1}$ and $\ell_{2}$ is equivalent to $f_{2}$. In this step, we further convert the two FDDs $f_{1}$ and $f_{2}$ to another two FDDs $f_{1}{ }^{\prime}$ and $f_{2}{ }^{\prime}$ such that the following three conditions hold: 1) $f_{1}$ is equivalent to $f_{1}{ }^{\prime}$;2) $f_{2}$ is equivalent to $f_{2}{ }^{\prime}$; and 3$) f_{1}{ }^{\prime}$ and $f_{2}{ }^{\prime}$ are semi-isomorphic. Two FDDs are semi-isomorphic if and only if they are exactly the same except the labels of their terminal nodes [8]. The algorithm for equivalently converting two FDDs to two semi-isomorphic FDDs is described in [8]. Fig. 3(c) shows the two semi-isomorphic FDDs converted from the two FDDs in Fig. 3(b).

3) Step III-FDD Logical AND/OR Operations: In previous steps, we equivalently convert two given ACLs $\ell_{1}$ and $\ell_{2}$ to two semi-isomorphic FDDs $f_{1}{ }^{\prime}$ and $f_{2}{ }^{\prime}$. In this step, we compute accept $\left(\ell_{1}\right) \cap$ accept $\left(\ell_{2}\right)$ and accept $\left(\ell_{1}\right) \cup \operatorname{accept}\left(\ell_{2}\right)$ using $f_{1}{ }^{\prime}$ and $f_{2}{ }^{\prime}$.

For any two semi-isomorphic FDDs $f_{1}{ }^{\prime}$ and $f_{2}{ }^{\prime}$, we define $f_{1}{ }^{\prime} \wedge f_{2}{ }^{\prime}$ as a new FDD $f$ such that $f$ is semi-isomorphic to $f_{1}{ }^{\prime}\left(\right.$ and $f_{2}{ }^{\prime}$ ) and a terminal node in $f$ is labeled 1 if and only if the two corresponding nodes in $f_{1}{ }^{\prime}$ and $f_{2}{ }^{\prime}$ are both labeled 1 (otherwise is labeled 0 ). This implies that the accept paths of $f_{1}{ }^{\prime} \wedge f_{2}{ }^{\prime}$ are the intersection of the set of accept paths in $f_{1}{ }^{\prime}$ and that in $f_{2}{ }^{\prime}$. Therefore, we can calculate accept $\left(\ell_{1}\right) \cap \operatorname{accept}\left(\ell_{2}\right)$ by calculating the accept paths in $f_{1}{ }^{\prime} \wedge f_{2}{ }^{\prime}$.

Similarly, for any two semi-isomorphic FDDs $f_{1}{ }^{\prime}$ and $f_{2}{ }^{\prime}$, we define $f_{1}{ }^{\prime} \vee f_{2}{ }^{\prime}$ as a new FDD $f$ such that $f$ is semi-isomorphic to $f_{1}{ }^{\prime}$ (and $f_{2}{ }^{\prime}$ ) and a terminal node in $f$ is labeled 0 if and only if the two corresponding nodes in $f_{1}{ }^{\prime}$ and $f_{2}{ }^{\prime}$ are both labeled 0 (otherwise is labeled 1). This implies that the accept paths of $f_{1}{ }^{\prime} \vee f_{2}{ }^{\prime}$ are the union of the set of accept paths in $f_{1}{ }^{\prime}$ and that in $f_{2}{ }^{\prime}$. Therefore, we can calculate accept $\left(\ell_{1}\right) \cup \operatorname{accept}\left(\ell_{2}\right)$ by calculating the accept paths in $f_{1}{ }^{\prime} \vee f_{2}{ }^{\prime}$. Note that after each FDD AND/OR operation, it is important to perform FDD reduction in order to bring down the FDD size expansion caused by the shaping procedure. An FDD is reduced if and only if it satisfies the following two conditions: 1) no two nodes are isomorphic; 2) no two nodes have more than one edge between them. Two nodes $v$ and $v^{\prime}$ in an FDD are isomorphic if and only if $v$ and $v^{\prime}$ satisfy one of the following two conditions: 1) both $v$ and $v^{\prime}$ are terminal nodes with identical labels; 2) both $v$ and $v^{\prime}$ are nonterminal nodes, and there is a one-to-one correspondence between the outgoing edges of $v$ and the outgoing edges of $v^{\prime}$ such that every pair of corresponding edges has identical labels and both edges point to the same node. An efficient FDD reduction algorithm that processes the nodes level by level from the terminal nodes to the root node using signatures to speed up comparisons is in [9]. We use an efficient FDD reduction algorithm that processes the nodes level by level from the terminal nodes to the root node using signatures to speed up comparisons. In our experiments, we have found that applying FDD reduction is critical in reducing memory usage for computing network reachability.

\section{Computing Path and FDD Matrices}

We next discuss the computing of two matrices, called path matrix and FDD matrix, which will be used in computing reachability matrices. The path matrix $P$ is an $n \times n$ matrix where each element $P[i, j]$ is the set of one-way paths from $N_{i}$ to $N_{j}$. For each path, letting $\ell_{1}, \ldots, \ell_{h}$ be the ACLs along the path, we compute the FDD that represents accept $\left(\ell_{1}\right) \cap \cdots \cap$ accept $\left(\ell_{h}\right)$. The resulting FDDs are stored in the FDD matrix $F$, which is also an $n \times n$ matrix.

First, we initialize matrices $P$ and $F$ as follows. For any $1 \leq$ $i, j \leq n$, if there is a path from $N_{i}$ to $N_{j}$ via a middlebox, then $P[i, j]$ consists of this path (which is composed of two links: the link from $N_{i}$ to the middlebox and the link from the middlebox to $N_{j}$, and $F[i, j]$ consists of the FDD that represent the intersection of the accept sets of the two ACLs associated with the two links; otherwise, $P[i, j]$ and $F[i, j]$ are both empty.

Second, we complete matrices $P$ and $F$ based on (8) and (9) using dynamic programming. We use $P[i, k] \circ P[k, j]$ to denote the set of paths where each path is a concatenation of a path in $P[i, k]$ and a path in $P[k, j]$. Similarly, we use $F[i, k] \wedge F[k, j]$ to denote the set of FDDs where each FDD is the logical AND of an FDD in $F[i, k]$ and an FDD in $F[k, j]$. Note that we remove 
all paths with cycles because cycles are typically prevented by routing protocols

$$
\begin{aligned}
& P[i, j]=\bigcup_{k \in \mathcal{N}} P[i, k] \circ P[k, j] \\
& F[i, j]=\bigcup_{k \in \mathcal{N}} F[i, k] \wedge F[k, j] .
\end{aligned}
$$

Third, for each FDD in $F[i, j]$, we reduce the domain of the source IP address field to the set of IP addresses used in subnet $N_{i}$ and the domain of the destination IP address field to the set of IP addresses used in $N_{j}$. We use $\operatorname{src}\left(N_{i}\right)$ to denote the set of packets whose source IP address is in $N_{i}$ and $\operatorname{dst}\left(N_{j}\right)$ to denote the set of packets whose destination IP address is in $N_{j}$. We use fdd $\left(\operatorname{src}\left(N_{i}\right)\right)$ to denote the FDD that represents $\operatorname{src}\left(N_{i}\right)$ and $\operatorname{fdd}\left(\operatorname{src}\left(N_{j}\right)\right)$ to denote the FDD that represents $\operatorname{dst}\left(N_{j}\right)$. Therefore, in this step, we replace each FDD $f$ in $F[i, j]$ by $\operatorname{fdd}\left(\operatorname{src}\left(N_{i}\right)\right) \wedge f \wedge \operatorname{fdd}\left(\operatorname{dst}\left(N_{j}\right)\right.$.

\section{Computing Reachability Matrices}

We are now ready to compute the six reachability matrices.

1) Reachability for Connectionless Protocols: For any $1 \leq k \leq|F[i, j]|$, we use $F[i, j]_{k}$ to denote the $k$ th FDD in $F[i, j]$ and $P[i, j]_{k}$ to denote the $k$ th path in $P[i, j]$. We use $\operatorname{Sub}_{\mathrm{CL}}\left(F[i, j]_{k}\right)$ to denote the UDP subtree of FDD $F[i, j]_{k}$. Recall that in computing reachability we choose protocol type to be the label of the root node. Therefore, the instantaneous reachability of the path $P[i, j]_{k}$ is

$$
A_{\mathrm{CL}}^{\mathrm{I}}[i, j, k]=\operatorname{Sub}_{\mathrm{CL}}\left(F[i, j]_{k}\right) .
$$

Accordingly, based on (4) and (5), the reachability upper bound and lower bound from $N_{i}$ to $N_{j}$ are calculated as follows:

$$
\begin{aligned}
& A_{\mathrm{CL}}^{\mathrm{U}}[i, j]=\operatorname{Sub}_{\mathrm{CL}}\left(\bigvee_{k=1}^{|F[i, j]|} F[i, j]_{k}\right) \\
& A_{\mathrm{CL}}^{\mathrm{L}}[i, j]=\operatorname{Sub}_{\mathrm{CL}}\left(\bigwedge_{k=1}^{|F[i, j]|} F[i, j]_{k}\right) .
\end{aligned}
$$

2) Reachability for Connection-Oriented Protocols: We first consider the case that all middleboxes on paths from $N_{i}$ to $N_{j}$ are stateful. We use $\operatorname{Sub}_{\mathrm{CO}}\left(F[i, j]_{k}\right)$ to denote the TCP/ICMP subtree. The instantaneous, upper-bound, and lower-bound reachability matrices are calculated using (2), (6), and (7) as follows:

$$
\begin{aligned}
A_{\mathrm{CO}}^{\mathrm{I}}[i, j, k] & =\operatorname{Sub}_{\mathrm{CO}}\left(F[i, j]_{k}\right) \\
A_{\mathrm{CO}}^{\mathrm{U}}[i, j] & =\operatorname{Sub}_{\mathrm{CO}}\left(\bigvee_{k=1}^{|F[i, j]|} F[i, j]_{k}\right) \\
A_{\mathrm{CO}}^{\mathrm{L}}[i, j] & =\operatorname{Sub}_{\mathrm{CO}}\left(\bigwedge_{k=1}^{|F[i, j]|} F[i, j]_{k}\right) .
\end{aligned}
$$

Second, we consider the case that all middleboxes on paths from $N_{i}$ to $N_{j}$ are stateless. As discussed in Section II-B.1, for the instantaneous reachability, we need to look at the reachability of each data path $P[i, j]_{k}$ and the corresponding signaling path $P[j, i]_{k^{\prime}}$. The swapping operator is implemented by func- tion $\operatorname{Swap}_{\mathrm{SD}}$. For an $\operatorname{FDD} f$, the function $\operatorname{Swap}_{\mathrm{SD}}(f)$ basically swaps the labels of source fields and destination fields. The instantaneous, upper-bound, and lower-bound reachability matrices are calculated using (3), (6), and (7) as follows:

$$
\begin{aligned}
& A_{\mathrm{CO}}^{\mathrm{I}}\left[i, j, k, k^{\prime}\right] \\
& =\operatorname{Sub}_{\mathrm{CO}}\left(F[i, j]_{k} \wedge \operatorname{Swap}_{\mathrm{SD}}\left(F[j, i]_{k^{\prime}}\right)\right) \\
& A_{\mathrm{CO}}^{\mathrm{U}}[i, j] \\
& |F[i, j]| \mid F[j, i] \\
& =\bigvee_{k=1} \bigvee_{k^{\prime}=1} A_{\mathrm{CO}}^{\mathrm{I}}\left[i, j, k, k^{\prime}\right] \\
& A_{\mathrm{CO}}^{\mathrm{L}}[i, j] \\
& =\bigwedge_{k=1}^{|F[i, j]| \mid F[j, i]} \bigwedge_{k^{\prime}=1}^{\mathrm{I}} A_{\mathrm{CO}}^{\mathrm{I}}\left[i, j, k, k^{\prime}\right] .
\end{aligned}
$$

For the case that the paths from $N_{i}$ to $N_{j}$ contain both stateful and stateless middleboxes, we use (13)-(15) to handle the stateful middleboxes and (16)-(18) to handle stateless middleboxes.

To compute the reachability for paths with IP tunneling based on (32) and (35), we need to use an FDD to represent $\Phi^{\mathrm{I}}(\pi, s)$ according to (31). Thus, we create two FDDs, namely "all-accept" FDD and "all-discard" FDD, to represent $\Sigma$ and $\emptyset$, respectively. The "all-accept" and "all-discard" FDDs contains five nodes (for 5-tuple packet) and five edges that are all labeled by a node's complete domain $(D(F(v)))$. The decision for "all-accept" FDD is 1 and for "all-discard" FDD is 0.

\section{Reachability Formulation With Packet TRANSFORMERS}

\section{A. Reachability Formulation With Nat}

Thus far, network reachability calculations are based on the assumption that packet header fields are not changed in the traversal from a source subnet to a destination subnet. Actually, there may be some packet transformers, such as NAT and PAT, on the intermediate middleboxes that modify packet headers. A NAT transformer on a middlebox may change the source address field of a packet from $x$ to $x^{\prime}$ and keep a record of this transformation in a table, which is used to change the destination field of the corresponding signaling packet from $x^{\prime}$ to $x$. A PAT transformer works similarly for port fields. Here, the path-coupled signaling assumption is necessary for paths that contain packet transforming filters. Next, we present reachability formulations for static and dynamic NAT.

1) Static NAT: Typically, a middlebox (such as a Cisco router [10] and IPtables [11]) applies NAT to a packet after it passes the ACL on the incoming interface and before it is sent to the ACL on the outgoing interface. Let middlebox $\gamma$ be the one on path $P_{i, j}(s)$ that uses a packet transformation (for source address or port number fields) function $T_{\mathrm{S}}: N_{i} \mapsto N_{i}{ }^{\prime}$, where $N_{i}{ }^{\prime}$ is the virtual subnet to which $N_{i}$ is mapped. We use $T_{\mathrm{S}}^{-1}$ to denote the reverse function. The instantaneous reachability for connectionless protocols is calculated using (1) as:

$$
\begin{aligned}
& R_{\mathrm{CL}}^{\mathrm{I}}\left(i, j, s, T_{\mathrm{S}}\right) \\
& \quad=\bigcap_{k=1}^{2 \gamma-1} A_{\mathrm{UDP}}\left(C_{k}\right) \bigcap T_{\mathrm{S}}^{-1}\left(\bigcap_{k=2 \gamma}^{2 M} A_{\mathrm{UDP}}\left(C_{k}\right)\right) .
\end{aligned}
$$


Note that applying function $T_{\mathrm{S}}^{-1}$ to $\bigcap_{k=2 \gamma}^{2 M} A_{\mathrm{UDP}}\left(C_{k}\right)$ means changing the source fields of every packet in $\bigcap_{k=2 \gamma}^{2 M} A_{\mathrm{UDP}}\left(C_{k}\right)$ from $N_{i}{ }^{\prime}$ to $N_{i}$.

The reachability bounds for connectionless protocols are calculated using (4) and (5) as follows:

$$
\begin{aligned}
& R_{\mathrm{CL}}^{\mathrm{U}}\left(i, j, T_{\mathrm{S}}\right)=\bigcup_{s \in \mathcal{S}} R_{\mathrm{CL}}^{\mathrm{I}}\left(i, j, s, T_{\mathrm{S}}\right) \\
& R_{\mathrm{CL}}^{\mathrm{L}}\left(i, j, T_{\mathrm{S}}\right)=\bigcap_{s \in \mathcal{S}} R_{\mathrm{CL}}^{\mathrm{I}}\left(i, j, s, T_{\mathrm{S}}\right) .
\end{aligned}
$$

For connection-oriented protocols, the middlebox $\gamma$ in the data path is the middlebox $\gamma^{\prime}$ in the signaling path (based on the path-coupled assumption). The instantaneous reachability formulation for data paths $R_{\mathrm{CO}}\left(i, j, s, T_{\mathrm{S}}\right)$ is as follows:

$$
\begin{aligned}
R_{\mathrm{CO}} & \left(i, j, s, T_{\mathrm{S}}\right) \\
& =\bigcap_{k=1}^{2 \gamma-1} A_{\mathrm{TCP}}\left(C_{k}\right) \bigcap T_{\mathrm{S}}^{-1}\left(\bigcap_{k=2 \gamma}^{2 M} A_{\mathrm{TCP}}\left(C_{k}\right)\right) .
\end{aligned}
$$

Similarly, the instantaneous reachability formulation for signaling path $R_{\mathrm{CO}}^{\leftarrow}\left(j, i, s, T_{\mathrm{D}}\right)$ is as follows:

$$
\begin{array}{r}
R_{\mathrm{CO}}^{\overleftarrow{ }}\left(j, i, s, T_{\mathrm{D}}\right) \\
=T_{\mathrm{D}}^{-1}\left(\bigcap_{k=1}^{2 \gamma^{\prime}-1} A_{\mathrm{TCP}}\left(C_{k}^{\prime}\right)\right) \bigcap_{k=2 \gamma^{\prime}}^{2 M^{\prime}} A_{\mathrm{TCP}}\left(C_{k}^{\prime}\right)
\end{array}
$$

where $T_{\mathrm{D}}$ transforms the destination addresses of signaling packets from $N_{i}$ to $N_{i}^{\prime}$.

Using (22), (23), and (3), we formulate instantaneous reachability for connection-oriented protocols as

$$
R_{\mathrm{CO}}^{\mathrm{I}}\left(i, j, s, T_{\mathrm{S}}, T_{\mathrm{D}}\right)=R_{\mathrm{CO}}^{\longrightarrow}\left(i, j, s, T_{\mathrm{S}}\right) \cap \tilde{R}_{\mathrm{CO}}^{\leftarrow}\left(j, i, s, T_{\mathrm{D}}\right)
$$

Note that (24) can be easily generalized to handle the paths that have multiple packet transformers.

The reachability bounds for connection-oriented protocols are formulated based on (6) and (7) as follows:

$$
\begin{aligned}
& R_{\mathrm{CO}}^{\mathrm{U}}\left(i, j, T_{\mathrm{S}}, T_{\mathrm{D}}\right)=\bigcup_{s \in \mathcal{S}} R_{\mathrm{CO}}^{\mathrm{I}}\left(i, j, s, T_{\mathrm{S}}, T_{\mathrm{D}}\right) \\
& =\bigcup_{s \in \mathcal{S}} R_{\mathrm{CO}}\left(i, j, s, T_{\mathrm{S}}\right) \\
& \bigcap \bigcup_{s \in \mathcal{S}} \tilde{R}_{\mathrm{CO}}^{\leftarrow}\left(j, i, s, T_{\mathrm{D}}\right) \\
& R_{\mathrm{CO}}^{\mathrm{L}}\left(i, j, T_{\mathrm{S}}, T_{\mathrm{D}}\right)=\bigcap_{s \in \mathcal{S}} R_{\mathrm{CO}}^{\mathrm{I}}\left(i, j, s, T_{\mathrm{S}}, T_{\mathrm{D}}\right) \\
& =\bigcap_{s \in \mathcal{S}} R_{\mathrm{CO}}\left(i, j, s, T_{\mathrm{S}}\right) \\
& \times \bigcap_{s \in \mathcal{S}} \tilde{R}_{\mathrm{CO}}^{\leftarrow}\left(j, i, s, T_{\mathrm{D}}\right)
\end{aligned}
$$

2) Dynamic NAT: Unlike in static NAT, an address $x$ in $N_{i}$ is dynamically mapped to an address $x^{\prime}$ in $N_{i}^{\prime}$ only when $x$ initiates a connection. Thus, the $T_{\mathrm{S}}$ function in dynamic NAT is not well defined. When a path contains a dynamic NAT transformer, computing the instantaneous reachability of the path is infeasible. Furthermore, the reachability bounds need to be formulated over both routing states and dynamic network address transformation. Therefore, the reachability upper bound from $N_{i}$ to $N_{j}$ in a network with dynamic NAT is defined as the set of packets that can traverse from $N_{i}$ to $N_{j}$ under $a$ viable routing state and $a$ NAT transformation. The reachability lower bound from $N_{i}$ to $N_{j}$ in a network with dynamic NAT is defined as the set of packets that can traverse from $N_{i}$ to $N_{j}$ underall routing states and all feasible NAT transformations.

Given the routing state $s$ and the transformation function $T_{\mathrm{S}}$, the instantaneous reachability for connectionless protocols is similar to static NAT and is calculated using (19). The reachability bounds for connectionless protocols, however, must include all possible NAT transformations. Thus, using (4), (5), and (19), the reachability bounds for connectionless protocols are calculated as follows:

$$
\begin{aligned}
& R_{\mathrm{CL}}^{\mathrm{U}}(i, j)=\bigcup_{s \in \mathcal{S}} \bigcup_{T_{\mathrm{S}} \in \mathcal{T}} R_{\mathrm{CL}}^{\mathrm{I}}\left(i, j, s, T_{\mathrm{S}}\right) \\
& R_{\mathrm{CL}}^{\mathrm{L}}(i, j)=\bigcap_{s \in \mathcal{S}} \bigcap_{T_{\mathrm{S}} \in \mathcal{T}} R_{\mathrm{CL}}^{\mathrm{I}}\left(i, j, s, T_{\mathrm{S}}\right)
\end{aligned}
$$

where $\mathcal{T}$ denotes all possible transformations on source fields.

For connection-oriented protocols, the instantaneous reachability is calculated similar to static NAT using (24) for a given $s, T_{\mathrm{S}}$, and $T_{\mathrm{D}}$. The reachability bounds for connection-oriented protocols are calculated based on (6), (7), (25), and (26) as follows:

$$
\begin{aligned}
& R_{\mathrm{CO}}^{\mathrm{U}}(i, j)=\bigcup_{s \in \mathcal{S}} \bigcup_{T_{\mathrm{S}}, T_{\mathrm{D}} \in \mathcal{T}} R_{\mathrm{CO}}^{\mathrm{I}}\left(i, j, s, T_{\mathrm{S}}, T_{\mathrm{D}}\right) \\
& R_{\mathrm{CO}}^{\mathrm{L}}(i, j)=\bigcap_{s \in \mathcal{S}} \bigcap_{T_{\mathrm{S}}, T_{\mathrm{D}} \in \mathcal{T}} R_{\mathrm{CO}}^{\mathrm{I}}\left(i, j, s, T_{\mathrm{S}}, T_{\mathrm{D}}\right) .
\end{aligned}
$$

\section{B. Reachability Formulation With IP Tunneling}

IP tunneling is achieved by encapsulating one IP packet inside another. It is commonly used in enterprise networks. For example, IPv6 packets can be carried in IPv4 tunnels in a network. In practice, IP tunnels are mostly used to send encrypted traffic from a private network to another over public networks. Popular tunneling protocols include Generic Routing Encapsulation (GRE) [12], a stateless IP tunneling protocol with transport protocol number 47, and Layer-2 Transport Protocol (L2TP), which operates on UDP protocol on port 1701 [13].

Next, we formulate network reachability on paths with IP tunnels. Suppose we have an IP tunnel between middleboxes $\gamma$ and $\gamma^{\prime}$ on path $P_{i, j}(s)$. As packets from $N_{i}$ to $N_{j}$ are encapsulated in IP packets with fixed source and destination addresses in the path from $\gamma$ to $\gamma^{\prime}$, if the tunnel from $\gamma$ to $\gamma^{\prime}$ can be established, then all packets from $N_{i}$ to $N_{j}$ can traverse from $\gamma$ to $\gamma^{\prime}$ inside the tunnel; otherwise, no packets from $N_{i}$ to $N_{j}$ can traverse from $\gamma$ to $\gamma^{\prime}$ inside the tunnel. We denote the tunnel reachability from $\gamma$ to $\gamma^{\prime}$ by $\Phi(\pi)$, where $\pi$ is the packet header for this IP tunnel. Thus, $\Phi(\pi)$ is the complete set of packet $\Sigma$ if the tunnel can be established and $\Phi(\pi)$ is empty if the tunnel cannot be established

$$
\Phi^{\mathrm{I}}(\pi, s)= \begin{cases}\Sigma, & \text { if } \pi \in \bigcap_{k=2 \gamma}^{2 \gamma^{\prime}-1} A\left(C_{k}\right) \\ \emptyset, & \text { otherwise. }\end{cases}
$$


Using instantaneous tunnel reachability, the instantaneous reachability for connectionless protocols is calculated using (1) as

$$
\begin{aligned}
R_{\mathrm{CL}}^{\mathrm{I}}(i, j, s, \pi) & =\left(\bigcap_{k=1}^{2 \gamma-1} A_{\mathrm{UDP}}\left(C_{k}\right)\right) \\
& \cap \Phi^{\mathrm{I}}(\pi, s) \cap\left(\bigcap_{k=2 \gamma^{\prime}}^{2 M} A_{\mathrm{UDP}}\left(C_{k}\right)\right) .
\end{aligned}
$$

Similar to (20) and (21), the reachability bounds for connectionless protocols are calculated as follows:

$$
\begin{aligned}
& R_{\mathrm{CL}}^{\mathrm{U}}(i, j, \pi)=\bigcup_{s \in \mathcal{S}} R_{\mathrm{CL}}^{\mathrm{I}}(i, j, s, \pi) \\
& R_{\mathrm{CL}}^{\mathrm{L}}(i, j, \pi)=\bigcap_{s \in \mathcal{S}} R_{\mathrm{CL}}^{\mathrm{I}}(i, j, s, \pi) .
\end{aligned}
$$

For connection-oriented protocols, suppose the tunnel identified by $\pi_{1}$ is between middleboxes $\gamma_{1}$ and $\gamma_{1}^{\prime}$ in the data path and the signaling path tunnel identified by $\pi_{2}$ is between middleboxes $\gamma_{2}$ and $\gamma_{1}$ and $\gamma_{2}^{\prime}$. Note that in IP tunneling the data paths and signaling paths can be decoupled. The instantaneous reachability formulation for data paths $R_{\mathrm{CO}}\left(i, j, s, \pi_{1}\right)$ and signaling paths $R_{\mathrm{CO}}^{\leftarrow}\left(j, i, s, \pi_{2}\right)$ are as follows:

$$
\begin{aligned}
& R_{\mathrm{CO}}\left(i, j, s, \pi_{1}\right)=\left(\bigcap_{k=1}^{2 \gamma_{1}-1} A_{\mathrm{TCP}}\left(C_{k}\right)\right) \\
& \cap \Phi^{\mathrm{I}}\left(\pi_{1}, s\right) \cap\left(\bigcap_{k=2 \gamma_{1}^{\prime}}^{2 M} A_{\mathrm{TCP}}\left(C_{k}\right)\right) \\
& R_{\mathrm{CO}}^{\leftarrow}\left(j, i, s, \pi_{2}\right)=\left(\bigcap_{k=1}^{2 \gamma_{2}-1} A_{\mathrm{TCP}}\left(C_{k}\right)\right) \\
& \cap \Phi^{\mathrm{I}}\left(\pi_{2}, s\right) \cap\left(\bigcap_{k=2 \gamma_{2}^{\prime}}^{2 M} A_{\mathrm{TCP}}\left(C_{k}\right)\right) .
\end{aligned}
$$

Using above formulas and (3), we formulate instantaneous reachability for connection-oriented protocols as

$$
R_{\mathrm{CO}}^{\mathrm{I}}\left(i, j, s, \pi_{1}, \pi_{2}\right)=R_{\mathrm{CO}}^{\longrightarrow}\left(i, j, s, \pi_{1}\right) \cap \tilde{R}_{\mathrm{CO}}^{\leftarrow}\left(j, i, s, \pi_{2}\right)
$$

Note that (35) can be easily generalized to handle the paths that have multiple IP packet tunnels.

Similar to (25) and (26), the reachability bounds for connection-oriented protocols are formulated as follows:

$$
\begin{aligned}
R_{\mathrm{CO}}^{\mathrm{U}}\left(i, j, \pi_{1}, \pi_{2}\right) & =\bigcup_{s \in \mathcal{S}} R_{\mathrm{CO}}^{\mathrm{I}}\left(i, j, s, \pi_{1}, \pi_{2}\right) \\
& =\bigcup_{s \in \mathcal{S}} R_{\mathrm{CO}}^{\longrightarrow}\left(i, j, s, \pi_{1}\right) \bigcap \bigcup_{s \in \mathcal{S}} \tilde{R}_{\mathrm{CO}}^{\overleftarrow{ }}\left(j, i, s, \pi_{2}\right)
\end{aligned}
$$

$$
\begin{aligned}
R_{\mathrm{CO}}^{\mathrm{L}}\left(i, j, \pi_{1}, \pi_{2}\right) & =\bigcap_{s \in \mathcal{S}} R_{\mathrm{CO}}^{\mathrm{I}}\left(i, j, s, \pi_{1}, \pi_{2}\right) \\
& =\bigcap_{s \in \mathcal{S}} R_{\mathrm{CO}}^{\rightarrow}\left(i, j, s, \pi_{1}\right) \bigcap_{s \in \mathcal{S}} \tilde{R}_{\mathrm{CO}}^{\leftarrow}\left(j, i, s, \pi_{2}\right) .
\end{aligned}
$$

\section{HANDLING PACKET TRANSFORMERS}

In this section, we present algorithms for computing reachability for networks that have packet transformation filters. There are two types of packet transformers, NAT and PAT. We use the terms private subnet and private addresses to refer to, respectively, the source subnet and its address pool, which is behind NAT middlebox. Similarly, we use the terms public subnet and public addresses to refer the virtual subnet and its address pool after NAT transformer, respectively. There are two types of NAT transformation: static NAT and dynamic NAT. A static NAT uses a static one-to-one mapping from private addresses to public addresses. This mapping table is configured by network administrators. A dynamic NAT maps private addresses to public addresses on-the-fly randomly. In PAT, all private addresses are mapped to a single public IP address but with different source port numbers, where each port number is designated for a connection initiated from the private subnet. Port address assignment is random.

In presenting our solution for handling packet transformers, we assume that all paths from one subnet to another need to go through the same packet transformer. This assumption is purely for the ease of presentation, and it imposes no limitation on our solutions for general cases. First, for paths that contain no packet transformers, we can easily compute reachability by combining the algorithms in this section and those in Section III. Second, our algorithms below can be easily extended to paths that contain more than one packet transformers by dividing each path into multiple chunks where each chunk contains only one packet transformer.

\section{A. Reachability for Connectionless Protocols}

We discuss reachability computation based on packet transformer types: static NAT, dynamic NAT, and PAT.

1) Static NAT: To handle static NAT, we only need to change the algorithm for computing an FDD matrix as follows. Given the $k$ th path from $N_{i}$ to $N_{j}$, which contains a static NAT that maps addresses in $N_{i}$ to $N_{i}^{\prime}$, we call the path from $N_{i}$ to the NAT middlebox a private subpath and the path from the NAT middlebox to $N_{j}$ apublic subpath. Let $f r$ and $f u$ be the FDDs that represent the set of packets that can be accepted by the ACLs on the private subpath and those on the public subpath, respectively. We still use $T_{\mathrm{S}}$ to denote the packet transformation function of the static NAT. Therefore, $\operatorname{src}\left(N_{i}\right) \wedge f r \wedge \operatorname{dst}\left(N_{j}\right)$ represents the packets that can traverse on the private subpath with source addresses in $N_{i}$, and $\operatorname{src}\left(N_{i}^{\prime}\right) \wedge f u \wedge d s t\left(N_{j}\right)$ represents the packets that can traverse on the public subpath with source addresses in $N_{i}^{\prime}$. Based on (19), the set of packets that can traverse from $N_{i}$ to $N_{j}$ passing the NAT can be calculated as follows:

$$
\begin{aligned}
F[i, j]_{k}=\left(\operatorname{src}\left(N_{i}\right)\right. & \left.\wedge f r \wedge d \operatorname{dst}\left(N_{j}\right)\right) \\
& \wedge T_{\mathrm{S}}^{-1}\left(\operatorname{src}\left(N_{i}^{\prime}\right) \wedge f u \wedge \operatorname{dst}\left(N_{j}\right)\right) .
\end{aligned}
$$

After this, reachability matrices can be computed using (10)-(12). Note that the operation $T_{\mathrm{S}}^{-1}\left(\operatorname{src}\left(N_{i}^{\prime}\right) \wedge f u \wedge\right.$ $\left.\operatorname{dst}\left(N_{j}\right)\right)$ basically replaces every source IP address $x^{\prime}$ in the domain of $N_{i}^{\prime}$ to $x$ in the domain of $N_{i}$ for FDD $\operatorname{src}\left(N_{i}^{\prime}\right) \wedge f u \wedge d \operatorname{st}\left(N_{j}\right)$.

2) Dynamic NAT: Consider all the paths from $N_{i}$ to $N_{j}$, which all pass through a dynamic NAT. Let FDDs $f r_{k}$ and $f u_{k}$ 
represent all the accepted packets by the $k$ th private and public subpaths, respectively, and let $a$ and $b$ be the total number of private and public subpaths, respectively. The upper-bound reachability of the private subpaths and public subpaths is calculated as follows:

$$
\begin{aligned}
f r^{\mathrm{U}} & =\bigvee_{k=1}^{a}\left(\operatorname{src}\left(N_{i}\right) \wedge f r_{k} \wedge \operatorname{dst}\left(N_{j}\right)\right) \\
f u^{\mathrm{U}} & =\bigvee_{k=1}^{b}\left(\operatorname{src}\left(N_{i}^{\prime}\right) \wedge f u_{k} \wedge \operatorname{dst}\left(N_{j}\right)\right)
\end{aligned}
$$

Similarly, lower-bound reachability of the private subpaths and public subpaths is calculated as follows:

$$
f r^{\mathrm{L}}=\bigwedge_{k=1}^{a}\left(\operatorname{src}\left(N_{i}\right) \wedge f r_{k} \wedge \operatorname{dst}\left(N_{j}\right)\right)
$$

Next, we compute the reachability bounds from $N_{i}$ to $N_{j}$ using $f r^{\mathrm{U}}, f r^{\mathrm{U}}, f r^{\mathrm{L}}, f u^{\mathrm{L}}$. First, we need to reorder the fields of FDDs $f u^{\mathrm{U}}$ and $f u^{\mathrm{L}}$ such that the label of the root is the source IP address. Because a dynamic NAT may map an address in $N_{i}$ to any address in $N_{i}^{\prime}$, to compute the reachability upper bound from $N_{i}$ to $N_{j}$, we need to disregard the source IP address field in $f u^{\mathrm{U}}$ by applying the logical OR operation on all the subtrees of the root. Thus, the upper-bound reachability for paths that contains a dynamic NAT transformer is calculated using (27) as follows:

$$
\begin{aligned}
& A_{\mathrm{CL}}^{\mathrm{U}}[i, j]=\mathrm{Sub}_{\mathrm{CL}} \\
& \quad \times\left(f r^{\mathrm{U}} \wedge \bigvee\left(\text { all subtrees of the root of } f u^{\mathrm{U}}\right)\right) .
\end{aligned}
$$

Similarly, to compute the reachability lower bound from $N_{i}$ to $N_{j}$, we need to disregard the source IP address filed in $f u^{\mathrm{L}}$ by applying the logical AND operation on all the subtrees of the root. Thus, the lower-bound reachability for paths that contains a dynamic NAT transformer is calculated using (28) as follows:

$$
\begin{aligned}
& A_{\mathrm{CL}}^{\mathrm{L}}[i, j]=\mathrm{Sub}_{\mathrm{CL}} \\
& \quad \times\left(f r^{\mathrm{L}} \wedge \bigwedge\left(\text { all subtrees of the root of } f u^{\mathrm{L}}\right)\right) .
\end{aligned}
$$

Note that reordering the packet fields of an FDD $f$ can be done in two steps. First, we generate an equivalent ACL $\ell$ from $f$ using the algorithms in [7]. Second, we construct an equivalent FDD $f^{\prime}$ from $\ell$ using the new packet field order.

For simplicity, we assume that a dynamic NAT transformer only changes the source IP address of a packet and does not change the source port field. However, even if it is the case, we can easily adapt our solution by excluding both fields from the FDDs $f u^{\mathrm{U}}$ and $f u^{\mathrm{L}}$.

3) PAT: In port address translation, the public addresses $\left(N_{i^{\prime}}\right)$ consist of a single IP address. Similar to dynamic NAT, PAT dynamically assign port numbers to new connections. Therefore, for paths with PAT, the instantaneous reachability cannot be computed because the transformation function is not well defined. However, we can define and compute reachability bounds for PAT similar to those for dynamic NAT. Formulas (39) and (40) are still valid, except that we reorder the packet fields of the FDDs $f u^{\mathrm{U}}$ and $f u^{\mathrm{L}}$ such that the source port field is the label of the roots.

\section{B. Reachability for Connection-Oriented Protocols}

As we stated previously, for simplicity, we present solutions to the case that all middleboxes on a path are stateful and the case that all middleboxes on a path are stateless. When all middleboxes on the path from $N_{i}$ to $N_{j}$ are stateful, for static NAT, we can use the FDD matrix calculated by (38) and use (13)-(15) to calculate the reachability matrices; for dynamic NAT, we can use (39) and (40) to calculate the reachability matrices except that the $\mathrm{Sub}_{\mathrm{CL}}$ function should be replaced by Sub $\mathrm{b}_{\mathrm{CO}}$. Next, we present solutions for the case that all middleboxes on a path are stateless based on the three types of packet transformers.

1) Static NAT: Considering the communication between $N_{i}$ and $N_{j}$ where all paths between them pass through a static NAT $t$, let $\overrightarrow{f r}, \overrightarrow{f u}, \overleftarrow{f u}, \overleftarrow{f r}$ be the FDDs that represent the packets that can traverse on the paths from $N_{i}$ to $t, t$ to $N_{j}, N_{j}$ to $t$, and $t$ to $N_{i}$, respectively. According to (38), the packets that can traverse on the data path are represented by $\overrightarrow{F[i, j]_{k}}=\left(\operatorname{src}\left(N_{i}\right) \wedge\right.$ $\left.\overrightarrow{f r} \wedge \operatorname{dst}\left(N_{j}\right)\right) \wedge T_{\mathrm{S}}^{-1}\left(\operatorname{src}\left(N_{i}^{\prime}\right) \wedge \overrightarrow{f u} \wedge \operatorname{dst}\left(N_{j}\right)\right)$. Similarly, the packets that can traverse on the signaling path are represented by $\overleftarrow{F[j, i]_{k}}=\left(\operatorname{src}\left(N_{j}\right) \wedge \overleftarrow{f r} \wedge d s t\left(N_{i}\right)\right) \wedge T_{\mathrm{D}}^{-1}\left(\operatorname{src}\left(N_{j}\right) \wedge \overleftarrow{f u} \wedge\right.$ $\left.\operatorname{dst}\left(N_{i}^{\prime}\right)\right)$. Therefore, according to (16), we can compute the instantaneous reachability between $N_{i}$ and $N_{j}$ as (41). The reachability bounds are calculated according to (17) and (18)

$$
A_{\mathrm{CO}}^{\mathrm{I}}\left[i, j, k, k^{\prime}\right]=\operatorname{Sub}_{\mathrm{CO}}\left(\overrightarrow{F[i, j]_{k}} \wedge \operatorname{Swap}_{\mathrm{SD}}\left(\overleftrightarrow{F[j, i]_{k^{\prime}}}\right)\right)
$$

2) Dynamic NAT: As discussed above, we use the new definitions for computing reachability bounds for networks with dynamic NAT. Let $a$ and $b$ be the total number of private and public paths, respectively. For the reachability upper bound of the data path from $N_{i}$ to $N_{j}$, we first compute the reachability upper bound for the private and public paths as follows:

$$
\begin{aligned}
& \overrightarrow{f r^{\mathrm{U}}}=\bigvee_{k=1}^{a}\left(\operatorname{src}\left(N_{i}\right) \wedge \overrightarrow{f r_{k}} \wedge \operatorname{dst}\left(N_{j}\right)\right) \\
& \overrightarrow{f u^{\mathrm{U}}}=\bigvee_{k=1}^{b}\left(\operatorname{src}\left(N_{i}^{\prime}\right) \wedge \bar{f} \overrightarrow{u_{k}} \wedge \operatorname{dst}\left(N_{j}\right)\right) \\
& \overleftarrow{f r^{\mathrm{U}}}=\bigvee_{k=1}^{a}\left(\operatorname{src}\left(N_{j}\right) \wedge \overleftrightarrow{f r_{k}} \wedge \operatorname{dst}\left(N_{i}\right)\right) \\
& \overleftarrow{f u^{\mathrm{U}}}=\bigvee_{k=1}^{b}\left(\operatorname{src}\left(N_{j}\right) \wedge \overleftarrow{f u_{k}} \wedge \operatorname{dst}\left(N_{i}^{\prime}\right)\right)
\end{aligned}
$$

Second, we reorder the packet fields for the FDDs $\overrightarrow{f u^{\mathrm{U}}}$ and $\overleftrightarrow{f u^{\mathrm{U}}}$ such that the root of $\overrightarrow{f u^{\mathrm{U}}}$ is labeled as the source IP address and the root of $\overleftarrow{f u^{\mathrm{U}}}$ is labeled as the destination IP address. Third, we compute the reachability upper bound as

$$
\begin{aligned}
& A_{\mathrm{CO}}^{\mathrm{U}}[i, j]=\operatorname{Sub} \mathrm{CO}\left(\overrightarrow{f r^{\mathrm{U}}} \wedge \bigvee\left(\text { all subtrees of the root of } \overrightarrow{f u^{\mathrm{U}}}\right)\right. \\
& \left.\wedge \operatorname{SwapsD}_{\mathrm{SD}}\left(\overleftarrow{f r^{\mathrm{U}}} \wedge \bigvee\left(\text { all subtrees of the root of } \overleftarrow{f u^{\mathrm{U}}}\right)\right)\right)
\end{aligned}
$$


For reachability lower bound, we first compute $\overrightarrow{f r} \overrightarrow{\mathrm{L}}, \overrightarrow{f u^{\mathrm{L}}}$, $\overleftarrow{f r^{\mathrm{L}}}$, and $\overleftarrow{f u^{\mathrm{L}}}$ as above by replacing all logical OR with Logical AND. Then, we compute the lower bound as follows:

$A_{\mathrm{CO}}^{\mathrm{L}}[i, j]=\operatorname{Sub}_{\mathrm{CO}}\left(\overrightarrow{f r^{\mathrm{L}}} \wedge \bigwedge\left(\right.\right.$ all subtrees of the root of $\left.\overrightarrow{f u^{\mathrm{L}}}\right)$ $\wedge \operatorname{Swap}_{\mathrm{SD}}\left(\overleftarrow{f r^{\mathrm{L}}} \wedge \bigwedge\left(\right.\right.$ all subtrees of the root of $\left.\left.\left.\overleftarrow{f u^{\mathrm{L}}}\right)\right)\right)$

3) PAT: We define and compute reachability bounds for PAT similar to those for dynamic NAT. Formulas (42) and (43) are still valid, except that we reorder the packet fields of the FDDs $\overrightarrow{f u^{\mathrm{U}}}$ and $\overrightarrow{f u^{\mathrm{L}}}$ such that the root is labeled the source port field and reorder the packet fields in $\overleftarrow{f u^{\mathrm{U}}}$ and $\overleftarrow{f u^{\mathrm{L}}}$ such that the root is labeled the destination port field.

\section{ONLINE REACHABILITY QUERIES}

After reachability matrices are calculated, we can use them as the engine for efficiently processing network reachability queries. In this section, we discuss languages for specifying reachability queries, ways of using such queries for network and security management, and algorithms for processing these queries. Based on the nature of queries, Quarnet supports three types of queries: upper-bound, lower-bound, and instantaneous. Upper-bound/lower-bound reachability queries are useful in verifying whether the ACLs on middleboxes satisfy certain security policies. Instantaneous reachability queries are useful for real-time security monitoring as the administrator identifies which paths are used at the time of querying. Such queries are also useful to verify whether the changes on the ACLs on some middleboxes have undesired impact on reachability. Based on the answer of queries, Quarnet supports two types of queries: closed and open. A closed query demands an answer of yes $/$ no. For instance, considering the network in Fig. 2, can all hosts in S1 communicate with Mail Server in S4 on TCP port 25 via any path? An open query demands an answer in terms of a set. For example, which hosts in S1 can access the Mail Server in S4 on TCP port 25 via any path from S1 to S4? As another example, what set of paths may let all hosts in $\mathrm{S} 1$ access the Mail Server in S4 on TCP port 25?

\section{A. Reachability Query Language}

SRQL Syntax: We define an SQL-like language called Structured Reachability Query Language (SRQL) for specifying reachability queries. SRQL has the following format:

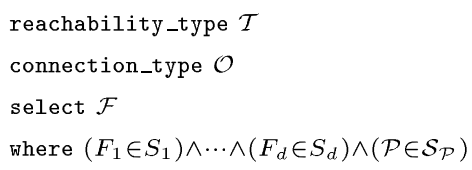

The reachability type $\mathcal{T}$ denotes the type of reachability, namely instantaneous $(I)$, upper-bound $(U)$, or lower-bound $(L)$. The connection type $\mathcal{O}$ denotes the connection orientation of transport protocols, namely connection-oriented $(C O)$ or connectionless $(C L)$. When the reachability type $\mathcal{T}$ is upper-bound or lower-bound, the select clause $\mathcal{F}$ is a subset of packet fields $\left\{F_{1}, F_{2} \ldots, F_{d}\right\}$; when $\mathcal{T}$ is instantaneous, $\mathcal{F}$ is a subset of fields $\left\{F_{1}, F_{2}, \ldots, F_{d}, \mathcal{P}\right\}$ where $\mathcal{P}$ denotes the attribute of "path." In the where clause, the predicate $\left(F_{1} \in S_{1}\right) \wedge \cdots \wedge\left(F_{d} \in S_{d}\right)$ specifies the set of packets that this query is concerned with and $\left(\mathcal{P} \in S_{P}\right)$ specifies the set of paths that this query concerns. For example, SRQL query for the question "Through what paths the mail server in S4 on TCP port 25 is accessible from S1?" is the following:

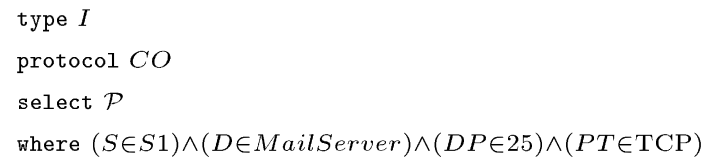

Note that we do not expect administrators to specify queries using SRQL directly. Instead, we expect a full-fledged implementation of Quarnet to provide a GUI for inputting queries and specifying paths. The SRQL will be used to formally represent a query under the hood.

SRQL Semantics: The result of an upper-bound reachability query, where $\mathcal{F}=\left\{\mathcal{F}_{1}, \ldots, \mathcal{F}_{h}\right\}$ and $\mathcal{F}_{i} \in\left\{F_{1}, F_{2}, \ldots, F_{d}\right\}$ for every $1 \leq i \leq h$, is defined as follows:

$$
\begin{aligned}
\left\{\left(\pi_{\mathcal{F}_{\infty}}, \ldots, \pi_{\mathcal{F}_{\zeta}}\right) \mid\right. & \left(\pi_{1} \in S_{1}\right) \wedge \cdots \wedge\left(\pi_{d} \in S_{d}\right) \text { and packet } \\
& \left(\pi_{1}, \ldots, \pi_{d}\right) \text { can traverse from its source } \\
& \text { to its destination at some time }\}
\end{aligned}
$$

The result of a lower-bound query is defined similarly, except that "at some time" is replaced by "at any time."

The result of an instantaneous reachability query, where $\mathcal{F}=$ $\left\{\mathcal{F}_{1}, \ldots, \mathcal{F}_{h}, \mathcal{P}\right\}$ and $\mathcal{F}_{i} \in\left\{F_{1}, F_{2}, \ldots, F_{d}\right\}$ for every $1 \leq i \leq$ $h$, is defined as follows:

$$
\begin{aligned}
\left\{\left(\pi_{\mathcal{F}_{1}}, \ldots,\right.\right. & \left.\pi_{\mathcal{F}_{h}}, \rho\right) \mid\left(\pi_{1} \in S_{1}\right) \wedge \cdots \wedge\left(\pi_{d} \in S_{d}\right) \text { and packet } \\
& \left(\pi_{1}, \ldots, \pi_{d}\right) \text { can traverse from its source to } \\
& \text { its destination through path } \left.\rho \text { where } \rho \in S_{P}\right\} .
\end{aligned}
$$

SRQL Example 1: We next give some query examples, where we use the shorthand $S$ for source IP, $D$ for destination IP, $S P$ for source port, $D P$ for destination port, and $P T$ for protocol type. The question "Can all hosts in $\mathrm{S} 1$ communicate with the mail server in S4 on TCP port 25?" can be formulated as the following query:

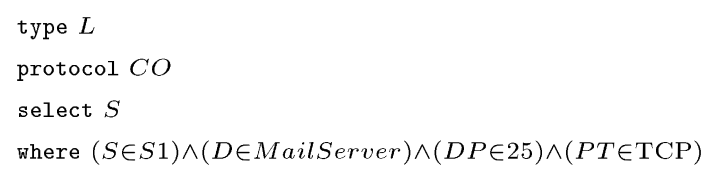

If the query result is all the IP addresses in S1, then the answer is "yes"; otherwise the answer is "no."

SRQL Example 2: SRQL query for the question "Through what paths the mail server in S4 on TCP port 25 is accessible from S1?" is the following:

$$
\begin{aligned}
& \text { type } I \\
& \text { protocol } C O \\
& \text { select } \mathcal{P} \\
& \text { where }(S \in S 1) \wedge(D \in \text { Mailserver }) \wedge(D P \in 25) \wedge(P T \in \mathrm{TCP})
\end{aligned}
$$


SRQL Example 3: The answer to some questions may be the union or intersection of multiple SRQL query results. For example, the answer for the question "Which hosts in S1 can access the Mail Server in S4 on both UDP and TCP port 25 via any path from $\mathrm{S} 1$ to $\mathrm{S} 4$ ?" is the intersection of the results of the following two SRQL queries:

$$
\begin{aligned}
& \text { type } L \\
& \text { protocol } C O \\
& \text { select } S \\
& \text { where }(S \in S 1) \wedge(D \in \text { MailServer }) \wedge(D P \in 25) \wedge(P T \in \mathrm{TCP}) \\
& \text { type } L \\
& \text { protocol } C L \\
& \text { select } S \\
& \text { where }(S \in S 1) \wedge(D \in \text { MailServer }) \wedge(D P \in 25) \wedge(P T \in \mathrm{UDP})
\end{aligned}
$$

\section{B. Reachability Query Engine Construction}

Our reachability query engine consists of six FDDs representing the six reachability matrices. We compute the six FDDs as follows. For each of the four upper-bound/lower-bound reachability matrices, we apply the logical OR operation to all matrix elements, where each element is an FDD representing the reachability between two specific subnets. The resultant FDD over $d$ fields represents the upper-bound/lower-bound reachability between any two subnets. For each of the two instantaneous reachability matrices, we compute the two corresponding FDDs as follows. First, we reduce the two instantaneous reachability matrices to two-dimensional matrices by combining the FDDs for the various paths from a source to a destination into one FDD. To achieve this, we first add a new node labeled with a new attribute "path" to each FDD as the root whose outgoing edge is labeled with path IDs, and then apply the logical OR operation to all FDDs regarding the reachability from one subnet to another. It is trivial to label every path with a unique ID. Second, for each of the two resultant two-dimensional matrices, we apply the logical OR operation to all elements and get an FDD over $d+1$ fields. The six FDDs will be used to process SRQL queries.

\section{Online Reachability Query Processing}

Reachability queries can be quickly processed by traversing one of the six FDDs computed above. The algorithm is essentially the same as the one described in [14] for querying one firewall policy.

\section{DISCUSSION}

\section{A. Complexity Analysis}

For a given network, let $n$ be the number of subnets, $\tau$ be the length of the longest path, $p$ be the number of the paths in the network, $g$ be the maximum of number of rules in an $\mathrm{ACL}$, and $d$ be the number of fields in each rule. Note that $d$ is typically a constant, which is 4 or 5 for IP networks. The complexity of constructing the equivalent FDD from an ACL with $g d$-dimensional rules is $O\left(g^{d}\right)$ [8]. The complexity of shaping the two FDDs constructed from two ACLs is $O\left((2 g)^{d}\right)=O\left(g^{d}\right)$. Therefore, the complexity of computing reachability matrices is $O\left(\tau \cdot p \cdot g^{d}\right)$. As the length of the paths cannot exceed a constant (i.e., 30), the complexity turns into $O\left(p \cdot g^{d}\right)$.

In theory, the total number of paths $p$ is exponential in terms of the number of subnets and middleboxes in the network. However, in practice, $p$ is much smaller than its theoretical upper bound because networks are typically designed following the hierarchical network design model [15]. Using this model, a network is designed in three layers, namely a core layer, a distribution layer, and an access layer. Security policies are mostly applied on the distribution layer, and the core layer is mainly used to facilitate routing between subnets. For networks designed by this model, the number of paths between two subnets is typically small (often one), and the length of a path is typically small.

\section{B. Recalculation for Incremental Changes}

As a network evolves over time, we need to update reachability matrices accordingly. There are three types of network changes that will affect reachability matrices: topology changes, configuration changes, and ACL changes.

Network Topology Changes: Any modification to the physical structure of a network, such as adding/removing a link, leads to network topology change. When such changes happen, we need to recompute the path matrix. For the new paths in the new path matrix, we need to recompute the corresponding elements in the FDD matrix $F$. The reachability matrices need to be updated accordingly. Hence, the complexity is $O\left(\tau \cdot p^{\prime} \cdot g^{d}\right)$, where $p^{\prime}$ is the number of new paths.

Subnet Configuration Changes: Network configuration changes refer to the changes in the subnet addresses or subnet masks. For such changes, the third step in computing the FDD matrix needs to be performed for the paths whose beginning or ending subnets are changed. Hence, the complexity is $O\left(\tau \cdot p^{\prime} \cdot g^{d}\right)$, where $p^{\prime}$ is the number of paths to and from the changed subnets.

Middlebox ACL Changes: ACL changes refer to the changes on ACL rules. When an ACL on a link changes, for all the paths that contains the link, we need to recompute the corresponding element in the FDD matrix. Hence, the complexity is $O\left(\tau \cdot p^{\prime}\right.$. $\left.g^{d}\right)$, where $p^{\prime}$ is the number of paths that contains the changed ACL.

\section{Integrating Network Routing for Real-Time Reachability}

To effectively monitor real-time network reachability, the network routing can be integrated with the instantaneous reachability matrix. If the Quarnet server collects routing states from the middleboxes once they change, it can identify the real-time reachability between all pairs of subnets. Moreover, by corresponding time to the network reachability the administrator can better understand the dynamics of temporal network outages or security breaches. He can also generate real-time queries to troubleshoot ongoing reachability problems.

\section{EXPERIMENTAL RESULTS}

\section{A. Reachability Computation}

We implemented Quarnet in $\mathrm{C}++$ and evaluated it on a university campus network. We focused the measurement on the execution time and memory usage of Quarnet. We concluded that Quarnet is sufficiently efficient to be used in practice as offline computation, although computing network reachability is a resource-consuming task in nature. 
TABLE II

RESUlTS ON COMPUTING REACHABILITY MATRICES

\begin{tabular}{|l|l|l|l|}
\hline & $\begin{array}{l}\# \text { of } \\
\text { FDDs }\end{array}$ & $\begin{array}{l}\text { Time } \\
\text { (mins) }\end{array}$ & $\begin{array}{l}\text { RAM } \\
(\mathrm{MB})\end{array}$ \\
\hline FDD construction & 192 & 2.1 & 1276 \\
\hline One-hop path calculation & 96 & 0.3 & 106 \\
\hline FDD matrix calculation & 2352 & 11 & 1505 \\
\hline $\begin{array}{l}\text { Reachability matrices calculation } \\
\text { for connection-less protocols }\end{array}$ & 2352 & 661 & 2400 \\
\hline $\begin{array}{l}\text { Reachability matrices calculation } \\
\text { for connection-oriented protocols }\end{array}$ & 2352 & 1 & 800 \\
\hline Total execution & 7344 & 675 & 4700 \\
\hline
\end{tabular}

This campus network consists of 48 subnets interconnected in a hierarchical topology. We model the core campus network as one subnet as most ACLs are deployed on edge routers/firewalls. We disregard the ACLs deployed on the core routers in the network because we have no access to them. Furthermore, their potential impact on the reachability calculation is expected to be small because the ACLs on core routers are typically very small and they mostly specify a few rules allowing only administrators to access the middleboxes for management purposes [15]. One subnet may contain multiple VLANs, and there are no ACLs among VLANs. For example, a VLAN could be the network that consists of all the printers in the subnet of a department. Note that we model the outside Internet as one subnet. This network does not have NATs/PATs or IP tunnels. Furthermore, all ACLs that we obtained are in use on stateful firewalls. In this network model, there are 49 subnets that are connected by 192 links through 2401 paths. Also, the total number of VLANs is 399 that are protected by 98 ACLs, where each ACL contains 573 rules in average (total number of rules: 56189 ). Among the 98 ACLs, 14 of them are original, and the rest are generated based on the statistical features of the original ones and the subnet addresses because we do not have access to all the ACLs in deployment.

We conducted experiments on a desktop computer with a Dual-Core AMD64 CPU $2.4 \mathrm{GHz}$ and $16 \mathrm{~GB}$ of RAM. This is a public machine running processes from other users as well. Our experimental results are shown in Table II. Note that the total amount of memory used by Quarnet is $4.7 \mathrm{~GB}$, not the sum of the memory used in each step because some memory is released after each intermediate step.

We gained two insights from our experiments. First, the running time of our algorithm goes up as the number of VLANs increases. This is because more number of VLANs means more intervals on the outgoing edges of nodes labeled with source or destination fields, which further means more edge splitting and subtree copying in performing FDD shaping. Second, the time for computing reachability matrices for connection-oriented protocols goes down dramatically as the number of stateful firewalls increases. This is because based on (13)-(15), we can use the FDDs calculated in the connectionless matrices and simply change the subtree functions from $\mathrm{Sub}_{\mathrm{CL}}$ to $\mathrm{Sub}_{\mathrm{CO}}$.

\section{B. Experimental Results Validation}

To validate the reachability matrices computed by Quarnet, we designed an ACL simulator that makes the decision for each packet by sequentially comparing the packet to every rule in an ACL. We generated a large number of packets for every path including all corner case packets based on the bounding values in

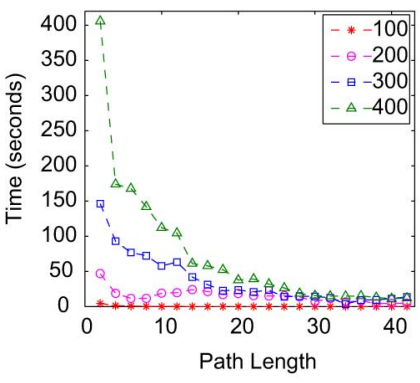

(a)

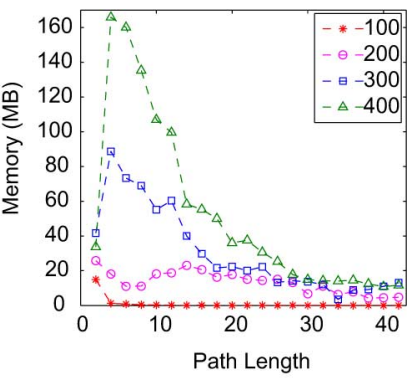

(b)
Fig. 4. Performance of Quarnet core operations. (a) Running time. (b) Memory usage.

the ACLs. We compared the decisions made by the ACL simulator and those made by the reachability matrices computed by Quarnet. The results are all positive.

\section{Performance of Core Operations}

The core operations in reachability computation are FDD logical AND and OR operations, the performance of which is the same as they all come down to FDD shaping. We are interested in evaluating their performance because it helps us to estimate the running time and memory usage of reachability matrix calculation. To evaluate the performance of the core operations, we created synthetic rules generated based on the statistical features of the real rules that we have obtained, such as the probability of unique IP addresses and port numbers, the decision bias of the rules, and the probability of fields being any. We focused on measuring the time and memory for calculating the reachability of paths with different lengths.

Fig. 4(a) and (b) shows the running time and memory usage of the core operations over different path lengths for a network with 100 subnets. The average number of rules per ACL ranges from 100 to 400 with $10 \%$ STD based on normal distribution. Note that based on the dynamic programming in Section III-C, the FDD for each path is calculated using a single logical operation. For instance, the FDD for a path of length 8 is calculated by an AND operation of either two FDDs, where each is for a path of length 4, or two FDDs, one for a path of length 6 and one for a path of length 2 . For any path length, we calculate the average cost of all possible permutations by which the FDD of the path may be calculated.

The interesting observation in Fig. 4 is that in general the running time and memory usage decrease as the path length increases. This makes sense because the rules in different ACLs from one network often share common fields. The source and destination IP address fields of many rules in the ACLs from one network are drawn from the IP prefixes of the subnets in the network. Similarly, many of the port number and protocol fields are drawn from the set of services provided by the network. When we shape two FDDs, each corresponding to a long path, because each of the two FDDs has gone through much edge splitting, the amount of new edge splitting tends to be small, which leads to reduced cost.

\section{Quarnet Scalability Analysis}

In this section, we evaluate the scalability of Quarnet by increasing the number of paths and rules on synthetic network topologies and synthetic ACL rules generated. We trained our 


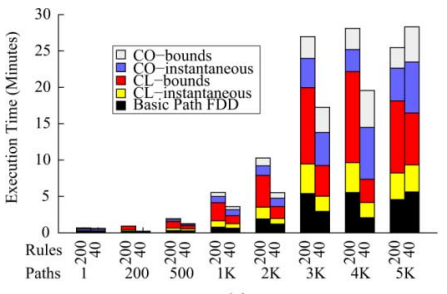

(a)

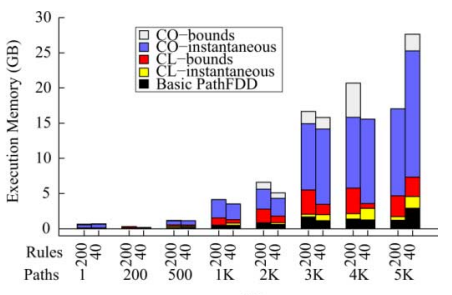

(b)
Fig. 5. Performance of Quarnet for synthetic networks. (a) Running Time. (b) Memory Usage.

rule generation model using real network topologies and configurations to generate close-to-reality networks. We use a largescale enterprise network with eight middleboxes (i.e., routers with ACLs on their interfaces), which divide the network into 10 subnets. We change the network connectivity by adding and removing edges to change the number of paths from 100 to 5000 . We perform scalability tests twice for each network topology, where in each test we use 20 ACLs on random router interfaces with $40 \pm 10$ and $200 \pm 50$ rules. The results for reachability computation execution time and memory are shown in Fig. 5(a) and (b), respectively.

The results confirm the observations we had in previous sections. First, in most cases, the execution time and memory usage increase linearly when the number of rules and paths increases. However, at some point, the number of paths and rules does not have much impact because the FDDs for different paths are very similar, which in turn means that less number of edge splitting and subtree copying is required. Second, the execution time and memory can vary for different Quernet operations. While the computation of CL matrices takes more time, the computation of CO matrices takes more memory.

Note that by comparing the results for synthetic networks and real-life networks, we can observe a very large difference between computation time of real-life networks (i.e., approximately $11 \mathrm{~h}$ ) and the computation time of synthetic networks (i.e., approximately $30 \mathrm{~min}$ ). As mentioned, the reason is because the number of VLANs (i.e., prefixes) is considerably larger (399 versus 10), and the diversity of rules is much higher, which leads to larger FDDs with more edge splitting and subtree copying in FDD shaping.

We also examined NAT/PAT on synthetic networks. The results show a negligible difference with reachability computation with no NAT/PAT. The reason is, for instantaneous reachability, the only overhead in computation is relabeling edges, and for bounds, because FDD's subtrees are very similar, the overhead of FDD subtree shaping is trivial.

\section{E. Performance of Online Querying}

As our reachability query engine uses FDDs as its core data structures, we evaluated the performance of online query processing by performing randomly generated queries over large FDDs with millions of nodes. Our experimental results in Table III show that our online reachability query engine is very efficient. For example, over an FDD with 2 million nodes, which is similar to the size of the FDDs used in the online query engine built for the university campus network that we experimented, the average processing time for a query is $0.075 \mathrm{~s}$, although some queries (less than $1 \%$ ) take $2-3 \mathrm{~s}$. The
TABLE III

PERFormanCE OF ONLINE QUERY PROCESSING

\begin{tabular}{|l|l|l|}
\hline $\begin{array}{l}\text { FDD Size } \\
\text { (\# nodes) }\end{array}$ & $\begin{array}{l}\text { Average Query } \\
\text { Processing Time }\end{array}$ & Outliers \\
\hline 0.5 million & $0.032 \mathrm{~s}$ & $1 \%$ of queries: $0.5 \mathrm{~s} \sim 1 \mathrm{~s}$ \\
\hline 1 million & $0.049 \mathrm{~s}$ & $0.8 \%$ of queries: $0.8 \mathrm{~s} \sim 1.5 \mathrm{~s}$ \\
\hline 2 million & $0.075 \mathrm{~s}$ & $1 \%$ of queries: $2 \mathrm{~s} \sim 3 \mathrm{~s}$ \\
\hline
\end{tabular}

existence of some outliers is because some randomly generated queries may cause the engine to search through a large portion of the FDD.

\section{RELATED WORK}

Active probing tools, which actively test network reachability by sending probing packets and analyzing the response packets, are commonly used by network administrators. Such tools include ping and traceroute, which use ICMP echo request/ reply or ICMP time-exceed packets to test whether a host on target network is reachable. The use of such tools is limited because they cannot verify the reachability of UDP or TCP packets. There are tools such as NMAP and NESSUS that can test the reachability of UDP or TCP packets. However, such tools have significant limitations. First, they cannot perform comprehensive testing due to the amount of packets that have to be generated. Second, the test results of such tools are valid only for the routing state at the time that the testing is performed and may not hold afterwards due to the change of routing states over time. Third, such tools only show the open ports on which a server daemon is listening and does not reveal the open ports with no server listening on them at the time of testing. In comparison, our Quarnet is nonintrusive and comprehensive.

As little work has been done on static network reachability analysis, no concrete algorithm for computing network reachability has ever been proposed previously. Xie et al. presented a model of network reachability for instantaneous reachability and reachability bounds. However, we significantly go beyond Xie et al.'s work along three dimensions: 1) reachability modeling and formulation; 2) algorithms for computing reachability; 3) solutions for reachability queries. Our model differs from Xie et al.'s work in the following aspects. First, we model a network as a graph over both routers and subnets, while Xie et al. model a network as a graph over only routers. Thus, Xie et al.'s model is limited to describing the networks where each subnet connects to only one router, while our model does not have this limitation. Furthermore, we calculate reachability between two subnets, and they calculate reachability between two middleboxes. Second, we distinguish network reachability formulations based on both the properties of transport-layer protocols (namely connectionless and connection-oriented protocols) and the statefulness of routers/firewalls on every path, while Xie et al. did not. Third, our model addresses IP tunneling and three types of packet transformations: static NAT, dynamic NAT, and PAT, while Xie et al.'s model addresses only static NAT. Xie et al. gave no implementable algorithms for computing reachability and no solutions for reachability queries. Recently, in a poster paper [16], Zhang et al. proposed monitoring and verifying reachability in real time by computing instantaneous reachability. However, they provided no algorithm for computing the reachability from a source to a destination along a given path and no experimental results. 
Furthermore, they have the other limitations of Xie et al.'s work mentioned above.

Some effort has been made to use binary decision diagrams (BDDs) for reachability analysis [17], [18]. However, such work can only answer host-to-host queries and cannot answer subnet-to-subnet queries as we do in this paper. We choose FDDs instead of BDDs for several reasons. First, it is extremely difficult to swap the values of packet fields or rearrange packet fields in a BDD calculated over multiple ACLs. Note that our methods for performing these operations on FDDs, which requires generating rules from FDDs and reconstructing FDDs, cannot be applied to BDDs because generating rules from a BDD could easily lead to millions of rules as reported in [8]. Second, a reachability query engine built with BDDs can only process closed queries that demand a yes/no answer. In contrast, our reachability query engine built with FDDs can process both closed queries and open queries. Third, the reachability calculated by BDDs is not human readable. In contrast, every element in our reachability matrix is an FDD, which can be visualized for examination.

In [19], Mayer et al. proposed Fang, a firewall analysis engine. Fang supports limited queries (over 3-tuples), and each query is compared to every rule in every ACL along every path from a source to a destination, which is very inefficient. There is some work, which is orthogonal to ours, on detecting reachability problems caused by routing faults (e.g., the faults identified in [20]), instead of ACL misconfiguration (e.g., [21]). In [22], Ingols et al. proposed algorithms for creating attack graphs for a network. However, their focus is not on reachability computation. Our proposed work is complementary to [22].

\section{CONCLUSION}

We make four major contributions in this paper. First, we model and formulate network reachability considering the differences in connectionless and connection-oriented transport protocols, stateless and stateful middleboxes, as well as the presence and absence of various packet transformers. Second, we present algorithms for computing network reachability matrices. Third, we give solutions for expressing and processing reachability queries. Fourth, we implemented our algorithms and conducted experiments on a campus network. Results show that our offline reachability computation is practical and online query processing is very efficient.

\section{REFERENCES}

[1] D. Oppenheimer, A. Ganapathi, and D. A. Patterson, "Why do internet services fail, and what can be done about it?," in Proc 4th USENIX Symp. Internet Technol. Syst., 2003, vol. 4, p. 1.

[2] Z. Kerravala, "As the value of enterprise networks escalates, so does the need for configuration management," Enterprise Computing \& Networking, The Yankee Group Rep., 2004.

[3] in G. G. Xie, J. Zhan, D. A. Maltz, H. Zhang, A. Greenberg, G. Hjalmtysson, and J. Rexford, "On static reachability analysis of IP networks," Proc. IEEE INFOCOM, vol. 3, pp. 2170-2183, Mar. 2005.

[4] A. Wool, "A quantitative study of firewall configuration errors," Computer, vol. 37, no. 6, pp. 62-67, Jun. 2004.
[5] M. Stiemerling, H. Tschofenig, and C. Aoun, "NAT/firewall NSIS signaling layer protocol (NSLP)," draft-ietf-nsis-nslp-natfw-05, 2005.

[6] A. Klenk, P. Schlicker, R. Kuhne, A. Fessi, C. Fan, F. Dressler, and G. Carle, "Path coupled accounting mechanisms for all IP networks," in Proc. IEE Conf. $3 G$ Beyond, 2005, pp. 1-5.

[7] M. G. Gouda and A. X. Liu, "Structured firewall design," Comput. Netw., vol. 51, no. 4, pp. 1106-1120, Mar. 2007.

[8] A. X. Liu and M. G. Gouda, "Diverse firewall design," IEEE Trans. Parallel Distrib. Syst., vol. 19, no. 9, pp. 1237-1251, Sep. 2008.

[9] A. X. Liu, C. R. Meiners, and E. Torng, "TCAM razor: A systematic approach towards minimizing packet classifiers in TCAMs," IEEE/ACM Trans. Netw., vol. 18, no. 2, pp. 490-500, Apr. 2010.

[10] Cisco Systems, San Jose, CA, "NAT order of operation," 2008 [Online]. Available: http://www.cisco.com/en/US/tech/tk648/ tk361/technologies_tech_note09186a0080133ddd.shtml

[11] O. Andreasson, "IPtables Tutorial 1.2.2," 2006 [Online]. Available: http://www.frozentux.net/documents/iptables-tutorial/

[12] D. Farinacci, T. Li, S. Hanks, P. Traina, and D. Meyer, "Generic routing encapsulation (GRE)," RFC 2784, 2000.

[13] W. Townsley, A. Valencia, A. Rubens, G. Pall, G. Zorn, and B. Palter, "Layer two tunneling protocol (L2TP)," RFC 2661, 1999.

[14] A. X. Liu and M. G. Gouda, "Firewall policy queries," IEEE Trans. Parallel Distrib. Syst., vol. 20, no. 6, pp. 766-777, Jun. 2009.

[15] Cisco Systems, San Jose, CA, "Internetworking design basics," 2012 [Online]. Available: http://www.cisco.com/en/US/ docs/internetworking/design/guide/nd2002.html

[16] B. Zhang, T. S. E. Ng, and G. Wang, "Reachability monitoring and verification in enterprise networks," presented at the SIGCOMM 2008.

[17] E. Al-Shaer, W. Marrero, A. El-Atawy, and K. ElBadawi, "Network configuration in a box: Towards end-to-end verification of network reachability and security," in Proc. IEEE ICNP, 2009, pp. 123-132.

[18] Y.-W. E. Sung, C. Lund, M. Lyn, S. G. Rao, and S. Sen, "Modeling and understanding end-to-end class of service policies in operational networks," in Proc. ACM SIGCOMM, 2009, pp. 219-230.

[19] A. Mayer, A. Wool, and E. Ziskind, "Fang: A firewall analysis engine," in Proc IEEE Symp. Security Privacy, 2000, pp. 177-187.

[20] V. Paxson, "End-to-end routing behavior in the Internet," IEEE/ACM Trans. Netw., vol. 5, no. 5, pp. 601-615, Oct. 1997.

[21] R. Mahajan, D. Wetherall, and T. Anderson, "Understanding BGP misconfiguration," in Proc SIGCOMM, 2002, pp. 3-16.

[22] K. Ingols, R. Lippmann, and K. Piwowarski, "Practical attack graph generation for network defense," in Proc 22nd Annu. IEEE Comput. Security Appl. Conf., 2006, pp. 121-130.

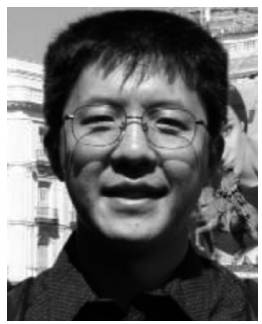

Alex X. Liu (M'08) received the Ph.D. degree in computer science from the University of Texas at Austin in 2006.

$\mathrm{He}$ is currently an Assistant Professor with the Department of Computer Science and Engineering, Michigan State University, East Lansing. His research interests focus on networking, security, and dependable systems.

Dr. Liu received the IEEE \& IFIP William C. Carter Award in 2004 and an NSF CAREER Award in 2009. He received the MSU College of Engineering Withrow Distinguished Scholar Award in 2011.

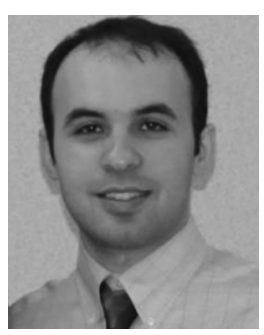

Amir R. Khakpour received the B.S. degree in electrical engineering from University of Tehran, Tehran, Iran, in 2005, and the M.Sc. degree in computer and communication networks from Telecom SudParis, Évry, France, in 2007, and is currently pursuing the Ph.D. degree in computer science and engineering at Michigan State University, East Lansing.

His research interests include Internet, network security, and large-scale systems. 